\title{
Orange is the new Black Box - Trump und Szenen der Herabsetzung
}

\author{
Lars Koch und Christina Rogers
}

„The White House Correspondents' Dinner is DEAD as we know it" war am 30. April 2018, 10:58 auf dem Twitter-Account @ realDonaldTrump zu lesen (Trump 2018a). Damit reagierte Trump auf eine kontrovers diskutierte Showeinlage der Comedienne Michelle Wolf, die beim jährlichen Gala-Dinner Personen des öffentlichen Lebens bloßgestellt hatte (C-Span 2018). Zum zweiten Mal in Folge nahm Trump nicht am Correspondents' Dinner teil und es bleibt abzuwarten, ob diese Leerstelle als symbolischer Ausdruck seiner aggressiven Distanz gegenüber den traditionellen Massenmedien Bestand haben wird.

Blickt man mit einigem Abstand auf die Aufregung vom April 2018, so wird deutlich, dass die damalige, affektiv aufgeladene Auseinandersetzung kaum etwas mit jenem Veranstaltungsformat an sich zu tun hatte. Die Vehemenz der Reaktion Trumps, die in gewohnter Manier als Breaking News auf CNN und den anderen Nachrichtensendern kursierte, steht in einem deutlichen Missverhältnis zur harmlosen Tradition des Correspondents' Dinner, das in der medialen Berichterstattung zuvor bloß eine Randnotiz darstellte: Trumps Twitter-Forderung „Put Dinner to rest, or start over!“ (30.04.2018, Trump 2018a) fügt sich ein in eine seit Jahren immer lauter werdende und nicht zuletzt aus den Reihen des Journalismus selbst kommende Kritik, die - allerdings aus anderer (nämlich diskursethischer) Motivation - den Spektakelcharakter des Dinner-Konzepts

\footnotetext{
L. Koch $(\triangle) \cdot$ C. Rogers

Dresden, Deutschland

E-Mail: lars.koch@tu-dresden.de

C. Rogers

E-Mail: christina.rogers@tu-dresden.de
} 
moniert. ${ }^{1}$ Über einige Lacher hinaus, die auf Kosten des kaum aus der Reserve zu lockenden Publikums gemacht würden, zeichne sich das Dinner, so die Kritik, einzig durch Belanglosigkeit aus (Patterson 2018). Der vorherrschende Eindruck, den die Beobachter*innen gewännen, sei der der Trostlosigkeit: „Washington people pretend to be glamorous in the basement of a characterless hotel, drinking bad wine, avoiding one another, and craning their necks to catch a glimpse of passing celebrities" (Rhodes 2018).

Dass Trump die Gelegenheit nutzt, mit der Geste moralischer Entrüstung den Tod des Correspondents' Dinners auszurufen, kann indes angesichts seiner rhetorischen Mobilmachung gegen die vermeintlichen Fake Media und der zum Ritual gewordenen Klage über das dekadente Establishment kaum verwundern. Bemerkenswert bleibt hingegen die konkrete Adressierung seiner Wut: So beschimpft Trump in seinen Tweets Wolf als eine „filthy ,comedian““, die es gewagt habe, seine Pressesprecherin Sarah Huckabee Sanders für ihr Aussehen anzugreifen (29.04.2018, 21:38, Trump 2018b). Zudem spricht er ihr jegliche Professionalität ab und stellt sie in eine Reihe mit Seth Meyers, der 2011 für die Showeinlage verantwortlich zeichnete und seither zu den Feinden Trumps zählt (ebd.). Darüber hinaus verweist der US-Präsident mit seinem Tweet auf das Jahr 2017, in dem das Correspondents' Dinner ebenfalls ein großer Reinfall gewesen sei. Die historische Reihe, die Trump aufmacht, ist kein Zufall, denn im Rahmen jener Dinner 2011, 2017 und 2018 wurde er am virtuosesten verspottet. Interessant ist dieser Zusammenhang in doppelter Hinsicht: Zum einen wird ersichtlich, dass Trumps prekäres Verhältnis zu den traditionellen Medien auf intrinsische Weise mit einer Szene der Herabsetzung verbunden ist, mit einer narzisstischen Kränkung, die sich im autoritären Charakter Trumps als Hass-Affekt fortwährend reaktualisiert. Zum anderen zeigt sich, dass Trumps übliche narrative Techniken zur Restabilisierung des eigenen Souveränitätsbegehrens hier nicht greifen: Mit Fake News-Unterstellungen, seinem bewährten Reaktionsmuster auf Kritik, kann man den Anwürfen des komischen Sprechakts offensichtlich nicht begegnen. Das Legitimations- und Abdichtungsnarrativ, das mit dem Fake News-Vorwurf verbunden ist, läuft im Falle des Humors ins Leere, weil letzterer immer eine mit alternativen Fakten, Überzeichnungen und Fehllektüren arbeitende, andere Wirklichkeit einer Sachlage oder Person entwirft,

\footnotetext{
${ }^{1}$ Die New York Times nimmt seit Jahren nicht mehr am Dinner teil, Vanity Fair und The New Yorker haben seit 2017 ihre Partys im Anschluss an die Gala abgesagt, zudem gab es Boykott-Aufrufe und Einladungen zu Gegenveranstaltungen (Grynbaum/Rogers 2017; Janker 2017; McCarthy 2017). Die Kontroverse 2018 betrifft folglich nur auf der Oberflächenebene den Auftritt von Michelle Wolf und Trumps Reaktion. Zugleich geht es auch um den sich wandelnden Charakter des Dinners, das angesichts der jährlich stetig wachsenden Zahl von eingeladenen Celebrities, Werbeschaffenden und Lobbyist*innen immer weniger dem ursprünglichen Gedanken eines informellen Kontakt- und Kommunikationsraums für Journalist*innen und Politiker*innen entspricht. Hinzu kommen prinzipielle Zweifel daran, ob die Nähe zwischen den demokratietheoretisch zu trennenden Sphären von Politik und Journalistik überhaupt wünschenswert ist.
} 
die in Spannung zur eigentlich hegemonialisierten Sicht tritt. Ein souveräner Umgang würde es erfordern, das ambivalente ,als-ob“ der Rede als punktuellen, karnevalesk eingehegten Aura-Verlust auffassen und aushalten zu können. Genau dazu ist Trump aber, wie der Eifer seiner Tweets zeigt, nicht in der Lage. Für ihn wird die Mikro-Krise der Autorität zu einem echten Angriff, weil er die gegen ihn gerichteten Invektiven nicht als Witze zum allgemeinen Amüsement des Publikums auffassen kann, sondern in ihnen Sprechakte erkennt, die seine Autorität prinzipiell anzweifeln. ${ }^{2}$

Angesichts der affektiven Dynamik, die die komische Kritik an Donald Trump auszulösen vermag, ist die Versuchung groß, sich mit psychoanalytischem Vokabular Trumps Psyche zu nähern und die angedeutete Narzissmus-These weiter zu entfalten. ${ }^{3}$ Anstatt aber vorschnell in den Chor von Beobachter*innen und Kommentator*innen einzustimmen, die das Phänomen Trump als Effekt einer deformierten Psyche zu verstehen versuchen und schaudernd imaginieren, was daraus für Gefahren für die Welt erwachsen, möchten die nachfolgenden Überlegungen eine andere, medienkulturwissenschaftliche Perspektive auf die Situation des Correspondents' Dinner werfen. Close Readings, die das Dinner als exemplarischen Schauplatz unterschiedlicher politisch-komödiantischer Phänomene begreifen, sollen einen genaueren analytischen Blick auf Affektdynamiken, Sicht- und Sagbarkeitsregeln der zeitgenössischen amerikanischen Politik sowie auf einige Aspekte der Medienkultur in den Zeiten Trumps eröffnen. Gefragt wird nach den veränderten Performanzen innerhalb des Felds der Politik. Dabei ist insbesondere der Normalisierung invektiver Rhetorik, also der Salon- und/oder Störfähigkeit beleidigender, beschämender, herabsetzender und feindkonstituierender Kommunikation im Kontext der Präsidentschaft Trumps nachzugehen. Es gilt, die gesellschaftlichen und kommunikativen Bedingungen herauszuarbeiten, die unterschiedliche und teilweise gegenläufige Muster in einem Erscheinungsraum herabsetzender Redeweisen zusammenbringen. Zur anvisierten Vermessung dieses Erscheinungsraums einer Politik der Herabsetzung gehört auch, zunächst intuitiv plausibel anmutende Unterscheidungen zu hinterfragen: Angesichts der Rhetorik Trumps scheint es naheliegend, eine sachliche und auf konsensuelle Problemlösung abzielende Kommunikation aufseiten der Demokratischen Partei zu verorten sowie eine Zeit vor Trump zu bemessen und dieser ,guten alten Zeit“ die Verrohung der Sitten und eine Logik der Feindschaft in der Ära Trump gegenüberzustellen. Anstelle einer solch dichotomischen Ordnung, auf deren Grundlage es in der medialen Berichterstattung erst möglich erscheint, Trump zum Great Disruptor der US-amerikanischen politischen Kultur zu stilisieren, wird

\footnotetext{
${ }^{2}$ Diese Positionierung gegenüber komödiantischen Reden hat sich mittlerweile über Trump hinausgehend so weit diskursiv in der Öffentlichkeit verfestigen können, dass im Jahr 2019 das Correspondents' Dinner mit seiner jahrzehntealten Tradition bricht: Anstatt einen Comedy Star für die zentrale Performance des Abends einzuladen, übernimmt der Historiker Ron Chernow die Rede (Heil/Farhi 2018; Grynbaum 2018).
}

${ }^{3}$ Zur Deutung Trumps als autoritärem Charakter im Sinne Erich Fromms vgl. Kellner 2016. 
vorgeschlagen, Trumps invective mode (vgl. die eher tentative philologische Konzeptionalisierung dieses Modus invektiven Sprechens bei Eisenhauer 2007: 15 ff) als sichtbarstes Ergebnis eines längerfristigen Prozesses zu sehen, in dem ganz unterschiedliche und einander wechselseitig verstärkende mediale, diskursive und politische Transformationen zum Tragen kommen. Die Correspondents' Dinners werden hier demnach als Mikrodramen beobachtet, in denen sich Diskursverschiebungen und Machtmechanismen innerhalb des öffentlichen, medialen und politischen Feldes nachzeichnen lassen. Trumps Verhältnis zu jenem Gala-Format soll als paradigmatische Konstellation rekonstruiert werden, an der sich jene Wahrheitsspiele und intermedial gerahmten Diskursphänomene nachvollziehen lassen, die mitgestalten, was im zeitgenössischen politischen Geschehen gesagt und worüber gelacht wird, wer den Ton angeben oder der symbolischen Exekution überantwortet werden kann und wer schließlich überhaupt in aufmerksamkeitsökonomisch relevanter Weise medial in Erscheinung tritt.

\section{"Well handled Sir, well handled"}

Im YouTube-Clip „C-SPAN: President Obama at the 2011 White House Correspondents' Dinner“ (C-Span 2011) ist zu sehen, wie - im Rahmen des Auftritts Obamas - den Anwesenden zunächst auf Bildschirmen ein Video gezeigt wird, in dem wehende amerikanische Flaggen leuchten, während eine Stimme den Satz „I am a real American" intoniert. Anschließend folgt eine Video-Collage US-amerikanischer Pop- und Politik-Ikonografie, die vom star-spangled-Banner über einen Weißkopfseeadler, Uncle Sam, Cowboys, Transformers, Karate Kid, Base Ball, Michael Jordan bis hin zu einem U-Boot reicht. Zu dieser Bildersequenz ist der patriotische Song „I am a real American“ von Rick Derringer zu vernehmen, der nicht nur als identitätspolitische Rahmung fungiert, sondern eine weitere popkulturelle Referenz in die Inszenierung einspeist, handelt es sich doch um den Theme-Song von Amerikas berühmtestem Wrestler Hulk Hogan. Das Leitmotiv der Collage, das den Zuschauer*innen in allen Übergängen entgegenspringt, ist ein Scan der offiziellen Langfassung der Geburtsurkunde Barack Obamas. Dieses audiovisuelle Intro gibt die Stoßrichtung für die nachfolgende präsidiale Performance vor: Eine ironisch-provokative Antwort auf all jene Vertreter*innen der sog. Birther-Bewegung, die in der aufgeheizten Atmosphäre des Wahlkampfs 2008/2009 und auch nach Obamas Amtsantritt keine Gelegenheit ausließen, den demokratischen Präsidenten als Fremdkörper in der weißen US-amerikanischen Kultur anzufeinden. Die Geburtsurkunde wird hier zum tragenden Element einer triumphalen Geste, die der politischen Rechten das Scheitern ihrer Diffamierungsstrategie vor Augen hält. Die Veröffentlichung der Langfassung von Obamas Geburtsurkunde wenige Tage vor dem Correspondents' Dinner war der Höhepunkt einer immer aggressiveren, protoparanoische Züge annehmenden Kampagne, die die Birthers um die rechtmäßige Staatsbürgerschaft des ersten Schwarzen 
Präsidenten organisiert hatten. ${ }^{4}$ Die Bewegung hatte es mit der Behauptung, Obama sei in Kenia oder Singapur geboren, geschafft, immensen Widerlegungsdruck auf die Demokratische Partei auszuüben. Wie für das Wahrheitsmanagement von Verschwörungstheorien üblich, wurden alle in den Medien präsentierten Indizien zu Obamas Herkunft von Hawaii und der damit verbundenen Rechtmäßigkeit seiner Präsidentschaft als Teil einer umfassenden Manipulation zurückgewiesen. Selbst Obamas Entscheidung von 2008, eine Kurzfassung seiner Geburtsurkunde $\mathrm{zu}$ veröffentlichen, reichte trotz der eindeutigen Stellungnahme der hawaiianischen Verwaltung nicht aus, um den Unterstellungen einen Riegel vorzuschieben. ${ }^{5}$ Angespornt durch eine Serie medial laut vernehmbarer Stimmen war es - zu Beginn des zweiten Wahlkampfs Obamas - kein anderer als Trump, der, inmitten kursierender Gerüchte um Pläne für eine eigene Kandidatur, den Zweifel an Obamas US-amerikanischer Staatsbürgerschaft erneuerte. Vor diesem Hintergrund richtete sich Obamas „I am a real American“-Collage, die live im US-Fernsehen übertragen wurde, nicht nur an die anwesenden Gäste der Gala, sondern an die gesamte Nation.

Entscheidend für die folgenden Überlegungen ist, in welcher Tonlage und mittels welcher kommunikativer Register das Intro auf die von dem Account @ realDonaldTrump getwitterten Mutmaßungen reagierte. Obama inszenierte ein komödiantisches Theater der Souveränität, das mit beißendem Spott den Anwesenden und der Öffentlichkeit vor Augen führte, von welch lächerlicher Sprecherposition aus Trump seinen Angriff führte. Die Geburtsurkunde war in der Performance nur der Startschuss zu einem Lehrstück über die gezielte Delegitimierung des politischen Kontrahenten, der - und das ist zentral - als Anwesender vor Publikum dem Verlachen und der Beschämung preisgegeben wurde: Nachdem die letzten Töne des Intros verklungen waren, verkündete Obama, an diesem Abend einen Schritt weiterzugehen und zum ersten Mal sein offizielles Geburtsvideo der Öffentlichkeit präsentieren zu wollen - woraufhin ein Zusammenschnitt aus Walt Disneys Der König der Löwen (1994) eingespielt wurde. Obamas Videoclip nutzt die paratextuelle Rahmung des Blockbuster-Materials, um eine Verschiebung vorzunehmen, die die Unterstellungen der Birther in einer ironischen Überaffirmation ad absurdum führt: Am unteren linken Bildrand ist „04 Aug. 1961 PM 7:24“ zu lesen, während im oberen rechten Bildrand ein roter Punkt neben „REC“ blinkt, was die Videosequenz an die Aufzeichnungsmuster von Amateurvideos rückbindet. Das Geburtsvideo präsentiert

\footnotetext{
${ }^{4}$ Zur begriffspolitischen Dimension der Groß- und Kleinschreibung „Schwarz/weiß“, vgl. Tißberger u. a. 2009.

${ }^{5}$ Hatte Aristoteles einst die berühmte Unterscheidung zwischen Geschichtsschreibung und Dichtung eingeführt, wonach Erstere das ,wirklich Geschehene mitteilt“, während es Letzterer darum geht, ,was geschehen könnte“, besteht eine Kernstrategie des Populismus darin, ebendiese Differenz in der Erwartungshaltung des Publikums aufzulösen. Besonders gut lassen sich die Verhältnisse verunklaren, wenn politisch nicht mehr entscheidend ist, ob etwas passiert ist, sondern nur noch, ob man glaubt, dass etwas passiert sein könnte oder passieren können wird (Aristoteles 2008: Kap. 9).
} 
nun die Sequenzen des Disney-Zeichentrickfilms, in denen der kleine Löwe Simba in den ostafrikanischen Pride Lands den versammelten Tieren als Thronfolger vorgestellt wird. Währenddessen ertönt die in Zulu gesungene Anfangssequenz von „The Circle of Life“ des Disney-Soundtracks.

Mit diesem Video vollzieht sich eine spielerische Souveränitätsgeste, die den Rassismus der Birther-Bewegung ebenso freilegt wie die Mechanismen und Engpässe innerhalb der Wahrheitsspiele rund um das Phänomen der Fake News. Im Sinne eines ironischen Reenactments aller Unterstellungen und Ressentiments fungiert das Video als eine Invektive, die ihre Schlagkraft dadurch erhöht, dass sie das Lachen der Anwesenden als Legitimationsressource für die eigene Version der Dinge nutzen kann. Das Video gewinnt sein provokatives Potenzial durch die Präsentation einer offensichtlichen Fiktion, die aber in der narrativen Neujustierung genau auf jene sozialen Fakten verweist, die für die rechts-reaktionären Birther nur schwer erträglich sind: Obama affirmiert die Vorwürfe, zum Upper Class America zu gehören, und geht sogar so weit, augenzwinkernd zuzugestehen, qua Geburt ein Royal zu sein - ohne seine Beziehung zu Kenia oder sein Black Heritage herunterspielen zu müssen. Anstatt immer wieder aus einer defensiven Position heraus Hawaii gegenüber Kenia stark zu machen, versetzt die Komikeinlage seine Geburtsstunde nach Afrika und lenkt damit die Aufmerksamkeit auf das, was Obama zu diesem Zeitpunkt ist: A Black leader living in the White House. Dabei verstärkt das tierische Personal des Videos die öffentliche Herrschaftsgeste noch zusätzlich, da diese Anspielung die Geschichte des Rassismus mit allen Zuweisungen des Primitivismus und der Nutzung von Tier-Analogien zur Herabsetzung von People of Color in Erinnerung ruft (Fanon 1981) und sie zugleich disidentifikatorisch und emanzipativ aneignet. ${ }^{6}$ Die ironische Anlage des Geburtsvideos wird dadurch auf die Spitze getrieben, dass ausgerechnet das Filmmaterial der Walt Disney Company verwendet wird, die - wie ihr Gründer und Namensgeber - wegen rassistischer Stereotypisierungen umstritten ist. Somit terrorisiert Obama die Birthers mit ihren eigenen Schreckgespenstern weißer Hegemonie - mehr noch: er spielt mit ihnen und macht sie zum Belustigungsobjekt der Zuschauer*innen.

\section{"Call Disney, if you don't believe me"}

Die Zweifel an der Kurzfassung der Geburtsurkunde waren einerseits rassistisch motiviert, speisten sich andererseits aber zusätzlich auch aus einem Problem, das sich im Zuge der Digitalisierung von Verwaltungsvorgängen eingestellt hatte: Kurzfassungen sind die offizielle elektronische Speicherungsart der Verwaltung,

\footnotetext{
${ }^{6}$ Disidentifikation ist ein Konzept das von José Esteban Muñioz unter Verweis auf Performances von queeren People of Color entwickelt wurde. Disidentifikation beinhaltet Wiederholungen und Differenzen als Kernelemente performativer Strategien, die ihre subversive Kraft aus der Verbindung aus präsentierter Identifikation und Abwehr ziehen (Muñioz 1999).
} 
allerdings verloren die staatlichen Dokumente im Zuge dieser digitalen Umwandlung ihren Abbild- und Referenzcharakter. Für einen nicht unerheblichen Teil der US-Bevölkerung verringerte sich damit das Vertrauen in das nationalstaatliche Archiv bzw. in dessen mediale Repräsentanz. Infolgedessen wurden sowohl die kurze wie auch die lange Version der Geburtsurkunde Obamas zum Anlass investigativer Untersuchungen interessierter Laien, die ihre vermeintlichen Indizien in den digitalen Verschwörungskosmos der Alt-Right einspielten. Um den von den Birthers bei jeder sich bietenden Gelegenheit wiederholten Manipulationsverdacht zu entkräften, musste die Langfassung des Dokuments, die eigentlich nur für die kommunale Verwaltung einsehbar war, der Öffentlichkeit zugänglich gemacht werden, wobei die Quantität (also die Länge des Dokuments) scheinbar die Qualität in Form der „Echtheit“ bestimmte. Anhand des Verlaufs der öffentlichen Debatte wird ersichtlich, wie die Information der Geburtsurkunde erst als In-formation evident wird, es also einer „Formenlehre der Macht“ bedarf, um dem Ziel der allgemein akzeptierten Beglaubigung näher zu kommen (Vogl 1998: 51). ${ }^{7}$ Die über Jahre gehegten medialen Zweifel und die Suche nach Beweisen für eine Manipulation gingen derweil mit einer wahren Material- und Medienschlacht einher: Plakatwände, Bumper Sticker, Kaffeetassen, E-Mails, Schlagzeilen, Postkarten und Schilder mit „Where's the Birth Certificate?“ wurden in Umlauf gebracht, während sich in Internetforen, auf Social Media-Plattformen, im Fernsehen und auf Pressekonferenzen der potenzielle Geburtsort Obamas jeweils unterschiedlich aktualisierte.

Vor dem Hintergrund dieser politisch interessierten Hermeneutik des Verdachts webt sich die Vorstellung des „,originalen“ Geburtsvideos während des Dinners in das aufgebotene mediale Arrangement affektiv ein: Das Dinner, wie es von Obama orchestriert wurde, wird dabei zu einer multimodalen Beglaubigungsshow. Um den Verdacht gegenüber der Authentizität und Aussagekraft der Geburtsurkunde als Trägermedium von Identität auszuräumen, kommt ein weiteres Medium das „Geburtsvideo“ - zum Einsatz. Dieses wird unter der Zeug*innenschaft des physisch anwesenden Publikums und der live über TV zugeschalteten Nation bei gleichzeitiger Anwesenheit seines menschlichen Referenten vorgestellt, um einen Zustand der Evidenz herzustellen. Parallel hierzu wird die Identitätsfeststellung via YouTube oder Twitter in den öffentlichen Diskurs als hegemoniale Deutung eingespeist. In einer protoparanoiden politischen Landschaft, in der Status und Legitimität von Fakten zunehmend umkämpft sind, scheint der diskursive Impact einer Behauptung primär nicht mehr von den Inhalten eines einzelnen

\footnotetext{
${ }^{7}$ Manche der öffentlichen Zweifel sind angesichts der Veränderungen, die Xerox Scanner in den digitalen Dokumenten erzeugen, nicht gänzlich unberechtigt, selbst wenn hinter den Angriffen mehrheitlich rassistische Motivationen stecken: Der Xerox-Skandal hat gezeigt, dass viele offizielle Dokumente tatsächlich durch die technischen Einstellungen von Kopierern und Scannern verfälscht sind. Bis heute halten sich Mutmaßungen, dass Obamas digitales Abbild der Geburtsurkunde dadurch fehlerhaft sei. Der Verdacht gegenüber Obama fällt hier mit einem Verdacht gegen die digitalen Medien zusammen (Kriesel 2014).
} 
Trägermediums abzuhängen. Entscheidender sind die möglichst komplexen, materiellen und medialen Verschränkungen, die sich um eine Sache, etwa um einen Geburtsort, bündeln. Die Frage „Where is the Birth Certificate?“" kann bei paranoider Lesart nicht einfach mit der Präsentation eines entsprechenden Schriftstücks beantwortet werden. Ebenso wenig bedeutet die Forderung „Show me your Papers" in dieser affektiven Dynamik ausschließlich eine reduktionistische Fixierung auf eine Staatsgewalt, die mit der Praxis des Racial Profiling eine prekäre Koalition eingeht. Vielmehr wird damit eine Anrufung artikuliert, in tatsächlich komplexer gewordene mediale und materielle Kopplungsprozesse einzutreten. ${ }^{8}$ In diesem identitätspolitischen Setting an das popkulturelle Gedächtnis einer filmbegeisterten Nation zu appellieren und staatliche Dokumente mit einem melodramatischen Blockbuster in Korrespondenz treten zu lassen, scheint so gesehen weniger absurd als der Rahmen eines Sketches zunächst suggeriert - das Rhizom hat den Stammbaum abgelöst. ${ }^{9}$ Konsequent endet diese "Aufklärungsarbeit" mit dem Satz: „Call Disney if you don't believe me. They have the original long-form version."

Später greift Obama diese Thematik wieder auf und adressiert explizit Trump, dem er eine große Hartnäckigkeit in der Ausschlachtung der Herkunftsfrage attestiert. Niemand anderes engagiere sich für die Beantwortung der zentralen Fragen der Nation derart entschlossen wie „The Donald“: „And that's because he can get back to focusing on the issues that matter, like: Did we fake the moon landing? What really happened in Roswell? And where are Biggie and Tupac?" Ohne sich weiter damit aufzuhalten, die wahnhaften Züge dieses Verdachtsdenkens zu explizieren, verweist Obama mit dem Nebensatz ,all kiddings aside“ polemisch auf Trumps Hemdsärmeligkeit im Umgang mit komplexen Entscheidungsnotwendigkeiten, wiederholt zu sehen etwa in der TV-Show Celebrity Apprentice, in der er Schauspieler*innen mit theatralischer Geste feuert. Nach einer kurzen rhetorischen Pause, in der es unter den Anwesenden zu Ausbrüchen von Gelächter kommt, wirft Obama dann ein: „And these are the kind of decisions that would keep me up at night. Well handled, Sir. Well handled." Die Reichweite dieser ironischen Aussage zeigte sich erst zwei Tage später, als bekannt wurde, dass Obama hinter den Kulissen des Dinners den Einsatz zur Tötung Osama Bin Ladens in Pakistan gestartet hatte. Das Correspondents' Dinner spielte sich also in einer realpolitischen Rahmung ab, die sowohl die fiktive Gewalt des „Bosses“ Trump und seine symbolische Macht, Menschen zu entlassen, wie auch Obamas eigene

\footnotetext{
${ }^{8}$ Obamas Performance lässt sich so betrachtet als ein Reparative Reading fassen, worunter Eve Kosofski Sedgwick den Versuch versteht, paranoide Lesarten in eine reparative Lesart zu überführen, die eher nach Verbindungen und Anknüpfungspunkten sucht als nach Offen- oder Freilegungen vermeintlich umfassender Wahrheiten (Sedgwick 1997).

${ }^{9}$ Gilles Deleuze und Félix Guattari stellen hierarchische und dichotomisch funktionierende Ordnungsmodelle, etwa Wissenshierarchien, die metaphorisch als Bäume mit Wurzeln und Ästen beschrieben werden, der Rhizomatik gegenüber. Wurzelgeflechte stehen hier eher für flexible Kopplungen, die sowohl re- als auch deterritorialisierend sind (vgl. Deleuze/Guattari 1993).
} 
scharfe Kritik an Trump einer Verhältnissetzung unterzog: Der eigentliche Situation Room lag woanders.

Obamas invektive Demontage war mit der Verspottung von Trumps Selbstbild als omnipotentem Geschäftsmann allerdings noch nicht zu Ende: In einer weiteren Collage präsentierte Obama die potenzielle Zukunft des White House unter einem kommenden Präsidenten Trump: Der offizielle Regierungssitz erscheint als eine Art Casino, an dessen Fassade mehrere Schriftzüge prangen: der Familienname „Trump“ und „the White House“ in einem leuchtend pinken 1980er-Jahre-Schriftzug sowie „Hotel, Casino, Golf Course and Presidential Suite“. Hinter den vergoldeten Säulen strahlt ein Kronleuchter, während auf dem vorderen Rasen zwei ältere Männer in Golfmontur stehen und Frauen in Bikinis Cocktails in einem Springbrunnen trinken. Vorlieben Trumps werden so als Inbegriff von Kitsch und schlechtem Geschmack ausgewiesen und als dem Präsidentenamt unangemessen gewertet. ${ }^{10}$ Trumps Stil, sein Geschmack, und - noch wichtiger - das Fundament seiner finanziellen wie gesellschaftlichen Stellung werden in Obamas Komposition zur Lachnummer. So schafft es Obama, Trumps Geltungs- und Anerkennungsanspruch ins Leere laufen zu lassen und seinen Gegner, dessen Themen und das Dinner selbst, in den eigenen Schatten zu stellen - wohl wissend, welche politischen Ereignisse um Bin Laden die Öffentlichkeit als eigentliche Nachricht dieses Tages erreichen werden.

Was hier vielleicht noch nicht absehbar war, ist, dass beide Ereignisse gemeinsam erinnert werden würden: Das Dinner als Szene der "traumatischen“ Beschämung Trumps und die Tötung des Al-Qaida-Anführers als Akt der kollektiven Traumabewältigung der vermeintlichen Free World. Als wäre Obamas Destruktionsarbeit noch nicht ausreichend gewesen, lieferte Seth Meyers im Fortgang des Abends weitere, noch bissigere Angriffe auf Trump.

In der C-Span-Live-Übertragung der Geschehnisse sind - begleitend zum schallenden Gelächter des Publikums - wiederholt Wechselschnitte zwischen dem starr am Tisch sitzenden Donald Trump und den am Pult vortragenden Barack Obama und Seth Meyers zu sehen. Die Aufnahmen kontrastieren jeweils Trump zu einem der Redner in einem Ungleichgewicht der Macht: der eine ist handlungsfähig als Sprecher, ermächtigt durch Mikrofone, TV-Übertragung oder Präsidentenamt, der andere erscheint als passiver Gast dem Spott der Anwesenden und des Fernsehpublikums ausgeliefert. Insofern wirkt Trump in diversen Szenen entmündigt und zu einem falschen Lächeln gezwungen. Von seinen Kontrahenten als jemand dargestellt, der unfähig ist, zwischen Fakten und Fiktionen zu unterscheiden, wird er in dieser Zeremonie der Statusdegradierung (Garfinkel 1956) als Lachfigur vorgeführt und dem beschämenden Blick der Nation preisgegeben.

Auch wenn das Correspondents' Dinner 2011 in seinem invektiven Sättigungsgrad kaum überschätzt werden kann, ist es nicht der psychologische Kern der politischen Karriere Trumps. Eine solche Erklärung des Phänomens Trump übersieht, dass das Verhältnis der Medienberichterstattung zum späteren US-Präsidenten seit

\footnotetext{
${ }^{10} \mathrm{Zu}$ Trumps Ästhetik und deren politischen Implikationen vgl. Seeßlen 2017.
} 
jeher von Polemik und voyeuristischer Entrüstungslust geprägt ist. Gleichwohl entwickelte die Traumatisierungs- und Rache-These gerade in den Wochen nach Trumps Wahlsieg eine große suggestive Kraft. Zu groß war die scheinbare Evidenz, als dass Nachrichtenagenturen und politische Kommentator*innen sich ihr auf der Suche nach Erklärungen für Trumps Bruch mit den Konventionen des politischen Betriebs hätten entziehen können. Diese Aufdeckungsmanie der Medien führte auch dazu, dass das Dinner 2011 immer wieder als eine zentrale Etappe auf Trumps Weg ins Weiße Haus dargestellt wurde. Die dokumentarische Netflix-Serie Trump: An American Dream (2017) etwa nutzt Trumps Beschämungserfahrung als dramaturgisches Kippmoment der Narration. In die gleiche Richtung zielen Analysen von Trumps Social Media-Verhalten, die für seine Tweets eine aggressivere Rhetorik, einen gesteigerten Willen zur invektiven Konfrontation und eine allgemeine Politisierung feststellen (Haberman/Burns 2016; Kolb 2016; Gopnik 2015; Porter 2017; Pierce 2016).

Das Correspondents' Dinner 2011 verdient hingegen vor allem Beachtung als Beispiel für die Formierung einer politischen Redeordnung, die sich den aufmerksamkeits- und resonanzökonomischen Bedingungen eines hybriden Mediensystems (Chadwick 2013) nach der Jahrtausendwende angepasst hat. So ist es keineswegs ein Zufall, dass Obamas Angriff auf Trump in Form einer komödiantischen Schmährede so nachhaltig zirkulierte. Obama erreichte eine souveräne Aussageposition gegenüber Trump, indem er die tradierte, in ihren Redeweisen gemäßigte Präsentationsform des politischen Amtes verließ. In einer Medienkultur, deren Aufmerksamkeits- und Valorisierungsordnung zunehmend von Beschleunigung und wertender Dichotomisierung geprägt ist (Reckwitz 2017; Pörksen 2018; Frevert 2017), kann vor allem auch eine solche politische Positionierung resonanzstark werden, die Konventionen der politischen Verständigungsverpflichtung stört, indem sie Kontrahent*innen herabsetzt und deren Anspruch auf Diskursteilhabe delegitimiert. Die Showeinlagen von 2011 stehen exemplarisch für einen Registerwechsel politischer Kommunikation, den Trump während seiner Präsidentschaft perfektionieren wird. Entscheidend an dieser Beobachtung ist, dass der inszenierte Registerwechsel der Correspondents' Dinner mit Trump zum alltäglichen Charakteristikum des POTUS geworden ist. Der 45. Präsident der USA ist für seine Wählerschaft nicht zuletzt deshalb so anziehend, weil sein Auftreten von einem Habitus robuster Virilität geprägt ist und er auch im Amt weiterhin auf Diffamierung und die beleidigende Verächtlichmachung seiner Gegner*innen setzt. Er folgt gängigen Kommunikationsmodi sozialer Netzwerke, Blogs usw. und löst immer wieder Shitstorms aus, die lawinenartig öffentliche und private Medien überrollen. Diese Form invektiver Rede ist als gängiges Stilmittel nicht nur in Netzkulturen und den medialen Kanälen der Alt-Right zu finden (Nagle 2018), sondern dringt von hieraus in immer größere Teile der bürgerlichen Mitte sowie der etablierten Politik ein. Auf diese Verschiebung der politischen Auseinandersetzung wird im Folgenden noch einmal zurückzukommen sein. 


\section{"And just a reminder to everyone. I'm here to make jokes"}

Obamas Correspondents' Dinner Speech ist diskursiv als besonders invektiver Sprechakt wahrgenommen worden, weil Trump zur selben Zeit im selben Raum und vor Zuschauer*innen - im Saal und vor den Empfangsgeräten - anwesend war. Gerade die Kopräsenz der Beteiligten verschärfte die agonale Situation. Dass das symbolische Verletzungspotenzial wie auch die „Körperkraft“ (Gehring 2007) einer invektiven Adressierung auch von der An- oder Abwesenheit der Beteiligten abhängt, lässt sich anhand der Skandalisierung der Comedy-Einlage von Michelle Wolf aus dem Jahr 2018 nachvollziehen (C-Span 2018). Wolf ist als scharfe feministische Kritikerin politischer Akteur*innen bekannt geworden und wurde nach ihrer Showeinlage insbesondere für ihre Ausführungen über Sarah Huckabee Sanders kritisiert, die während des Dinners in ihrer unmittelbaren Nähe saß. Wolf verglich sie mit dem Negativ-Charakter der Tante Lydia (Ann Dowd) aus der erfolgreichen TV-Serie The Handmaid's Tale (Hulu, seit 2017). Wolf bezeichnete Huckabee Sanders als einen „Uncle Tom“, womit sie darauf anspielte, dass Sanders als weiße Frau andere weiße Frauen enttäusche, weil sie sich mit der von Männern dominierten heterosexistischen Geschlechterordnung arrangiere und damit den status quo stütze. Darüber hinaus machte sich Wolf über den Namen ihres Gegenübers, Sanders politisches Auftreten und ihren lockeren Umgang mit der Wahrheit lustig: „Like she burns facts, and she uses that ash to create a perfect smoky eye.“ Wolfs Anspielungen wurden in Online-Foren und Nachrichtenkommentaren rückwirkend als entsolidarisierend und sexistisch bewertet. So wurde der Vergleich mit Tante Lydia nur als übergriffiger Verweis auf das körperliche Erscheinungsbild der nach dominierenden Schönheitsbildern „hässlichen“ Figur verstanden. Dass Wolf eigentlich einen metaphorischen Kommentar zur diskursiven Funktion von Huckabee Sanders innerhalb einer heteronormativen Ordnung im Sinn hatte, wurde übersehen. Ebenso blieb unhinterfragt, welcher sexistische Lookism der öffentlichen Empörungswelle zugrunde lag. Im gleichen körperzentrierten Register wurde auch der Scherz über die Herstellung von Huckabee Sanders' Schminke gelesen: Der Bezug zum interessierten Fakteneinsatz wurde ausgeblendet, einzig der wenig schmeichelhafte visuelle Vergleich wurde kritisiert. Der Sexismus, der Wolf unterstellt wurde, produzierte eine spezifische Formatierung ihrer Aussagen, insofern die sexistische Matrix die Öffentlichkeit für eine bestimmte Wahrnehmungs- und Rezeptionsweise ihres Humors prädisponierte: Wolfs invektive Sprechakte wurden im Feld visueller Körperlichkeit gerade nicht als politische Intervention verstanden und kommentiert. Stattdessen drehte sich die öffentliche Debatte um die Frage, ob es sittlich sei, sich als Frau derartig über andere Frauen zu äußern.

Auffällig an beiden Szenerien - Obama/Meyers/Trump 2011 sowie Wolf/ Huckabee Sanders 2018 - ist, dass sich die Skandalträchtigkeit der invektiven Rede nicht zuletzt aus der Interaktion unter Anwesenden ergibt. Die Relation zwischen Bühne und Publikum ist ein wichtiger Faktor, der das Spektrum des Möglichen reguliert. Die Rahmung des Gala-Dinners erlaubt nicht die gleiche eskalative Entgrenzung wie sie für die digitale Abwesenheitskommunikation der Shitstorms, Hasstiraden oder Trollings typisch ist. Demgegenüber ist Trumps Politik der 
Herabsetzung Teil eines neuen medialen Sagbarkeitsregimes, das kaum noch kommunikative Tabus kennt. In einer polarisierten Medienlandschaft und verbunden mit den technischen Möglichkeiten der many-to-many-Kommunikation findet sich immer ein Publikum, das zustimmend applaudiert, blogt, retweetet oder Likes verteilt. Werden Szenen der Beleidigung medial aufgezeichnet oder realisieren sie sich von vorneherein als Kommunikation unter räumlich Abwesenden qua der Aufschreibesysteme der sozialen Medien, so stellt sich sukzessive eine Normalisierung ein, die den politischen Gebrauch des Invektiven nicht nur gesellschaftsfähig macht, sondern sogar zur Ressource politischer Mobilisierung werden lässt.

Vor diesem Hintergrund ist es nur auf den ersten Blick paradox, dass gerade Trump sich über die „schmutzigen“ Angriffe auf seine Pressesprecherin ostentativ entrüstet. Und es wäre wohl auch unzutreffend, die Unmutsbekundungen Trumps als rein taktisches Kalkül zu werten. Vielmehr scheint er von einem moralischen Gefühl der Empörung angetrieben, das in den Kern der populistischen Gesinnungsgemeinschaft führt, in der er mit seinen Wähler*innen verbunden ist: Michele Wolf repräsentiert in seinen Augen jene liberale Doppelmoral des Establishments, die sich mit Redeverboten und Sexismusvorwürfen über andere erhebt, dabei jedoch vermeintlich die eigenen sexistischen Implikationen übersieht. Trumps Einwürfe stilisieren Huckabee Sanders zu einem Opfer diffamierender öffentlicher Rede, wie er es selbst im Rahmen des Dinners 2011 war. In diesem Stereotyp gehört Wolf zu jenen Karrierefrauen wie Hillary Clinton, die nicht nur die männlichen Privilegien bedrohen, sondern auch die Integrität anderer Frauen gefährden. So treffen Forderungen, feministische Frauen einzusperren „lock her up“ war ein Ruf vieler Trump-Anhänger*innen im Wahlkampf gegen Clinton -, auf Trumps „locker room talk“: Sie realisieren ein sexistisches Gefüge, das Frauen zu Objekten männlicher Verfügungsgewalt macht. Diese Situationsbeschreibung ist kennzeichnend für die argumentative Logik des Trumpismus und dessen Selbstviktimisierung, die mit der Diskursstrategie der Alt-Right korrespondiert.

Mit dem Ziel eines besseren Verständnisses der aktuellen Polarisierungstendenzen, die von manchen Akteur*innen schon als vermeintlicher ,Kulturkrieg' ausgedeutet werden, erscheint es produktiv, diese diskursive Funktion der Opfer-Position genauer in den Blick zu nehmen. Zu den rhetorischen Mitteln der Neuen Rechten in den USA wie in Europa zählt eine Argumentationsfigur, die die Verantwortlichkeit für die unterstellte Krise von Nation und Gesellschaft dem sog. Establishment zuweist und aus diesem Verschulden einen moralischen Anspruch auf Erneuerung und eine eigene Führungsrolle in einem notwendigen Prozess kultureller und politischer Reinigung ableitet. In der Tat zeigt sich hier eine neue Form neu-rechter Identiätspolitik, die eng mit der Klage über Redeverbote verbunden ist. Die Entrüstung über eine als gegeben erachtete Meinungszensur im Dienste ,politisch korrekter“ Sprache entfacht im aktuellen Kampf um Deutungshegemonie wechselseitig eine affektive Dynamik, ${ }^{11}$ die zur Norma-

\footnotetext{
${ }^{11}$ Zunächst in den 1980er Jahren benutzt, um Zustimmung zur Vermeidung beleidigender Sprachausdrücke zu signalisieren, wurde Political Correctness in den 1990er Jahren sukzessive zum diffamierenden Kampfbegriff der Neuen Rechten im metapolitischen Kampf um Deutungshegemonie (Hughes 2009).
} 
lisierung gewaltsamer Schmährede beiträgt und so als treibendes Moment der Umbildung der Sagbarkeitsordnung fungiert. Die Vehemenz und Herabsetzungsneigung rekurriert auf die Behauptung, von einer minoritären, diskriminierungsgefährdeten Position aus zu sprechen. Nicht nur das Establishment wird dadurch zur Angriffsfläche, sondern all jene anderen Minderheiten, die sich ebenfalls innerhalb von Anerkennungsdiskursen situieren. Die eigene Erregungsintensität wird dabei in einer verqueren Logik zum Beweis der bösen Intentionen des jeweils als Establishment markierten Gegners: Wut, Aggression und der Wille zur Herabsetzung erscheinen als legitim, weil die Eliten von Grund auf unmoralisch handeln und die Gesellschaft für die eigenen Zwecke missbrauchen. Auch die Correspondent's Dinners 2017 und 2018 können hier als Beispiel und Resonanzraum angesehen werden. So ließ sich der Komiker Hasan Minhaj beim Dinner 2017 etwa zu der Aussage hinreißen, die tradierten Nachrichtenmedien seien nunmehr eine neue Minorität, die um Gehör kämpfen müsse (ABC News 2017). Ein ähnliches Register zog auch Wolf 2018, wenn sie auf Trumps Herkunft aus dem Geldadel anspielte (C-Span 2018). Sie legte damit den Fokus auf Trumps überaus erfolgreiche Strategie, sich selbst als das moralisch untadelige Andere des Establishments zu inszenieren. Die nachstehenden Gedanken folgen diesem Ordnungsgefüge und legen dabei einen Fokus auf die zugrunde liegenden politischen Aufmerksamkeitsökonomien. Dabei geht es darum, die Prozesse und Logiken der Aneignung und Verwerfung, die Trumps Politik systematisch durchziehen, herauszuarbeiten.

\section{„Trump is so broke! How broke is he?"}

Obwohl Trump als Millionärssohn, Hotel- und Casinomogul, berühmter Entrepreneur und Teil der rich upper society zwischen New York und Atlantic City alles andere als ein blue-collar citizen ist, wurde schnell klar, dass die von ökonomischen und kulturellen Abstiegsängsten affizierte untere weiße Mittelschicht die Mehrheit seiner Wählerschaft ausmacht (Roswell/Diego-Rosell 2016). Trump kommt als volksnaher Dandy daher, der die Wünsche, Hoffnungen und Träume der ,ganz normalen“ Bürger*innen versteht. Das zentrale affektpolitische Motiv seiner populistischen Mobilisierungsstrategie ist die bei jeder Gelegenheit artikulierte Wut über die liberalen Eliten der Ost- und Westküste. Jene Eliten, die paradoxerweise zugleich die Kund*innen des Geschäftsmanns Trump sind, hätten zu den Menschen im Lande ein bloß parasitäres Verhältnis. Das Bündnis, das Trump so mit den Zurückgelassenen einer nur für wenige profitablen Ökonomie und Demokratie eingeht, fußt auf einer geteilten Affektion: Diese entspringt einerseits aus der Behauptung, durch die herrschende Klasse verraten worden zu sein, und überträgt sich andererseits in die mit Vehemenz vorgebrachte Forderung nach Sichtbarkeit und Gehör. In dieser affektiven Dynamik wird auch jegliche Komplizenschaft mit der etablierten Ordnung als Verrat angesehen. Der Anspruch, gegen das ,verdorbene“ politische System eine radikal andere Form zu setzen, mündet in ein Verhalten, das sich nicht in einen bisher gültigen Rahmen fügen darf. Der Veränderungsgrad wird damit zum Indikator der Ernsthaftigkeit der 
politischen Programmatik. Als Praxis einer systemkritischen Selbstpositionierung muss die symbolische Konfrontation mit der etablierten Redeordnung radikal sein. Tabus zu brechen und Medienskandale zu initiieren diskreditiert aus dieser Perspektive nicht die politische Handlung, sie macht sie vielmehr erst wahrhaftig. Der physische Körper wird dabei zu einem Medium der Beglaubigung: Beleidigen, Brüllen, eine unkontrollierte, ausschweifende Gestik usw. gehören ebenso zum Repertoire der Inszenierung von Unkonventionalität und Authentizität wie Schläge unter der Gürtellinie, Drohungen und Lügen. Trumps exzessiver Gebrauch von Twitter hat seinen Grund nicht nur in der Möglichkeit der Umgehung der Filterfunktion traditioneller Nachrichtenkanäle. Wichtiger noch ist der Umstand, dass Trump in der direkten Ansprache und in der Kommentierung des politischen Alltags - sei es als wütender Angriff, als kühler Liebesentzug oder als euphorisches Lob - einen Eindruck von Authentizität und Unangepasstheit erzeugen kann, der bei seiner Wählerschaft verfängt (vgl. Turner 2018: 147). Das „real““ in @ realDonaldTrump ist der Frame für alle seine Tweets.

Das neu-rechte Selbstverständnis, Teil einer antihegemonialen Graswurzel-Bewegung zu sein, dokumentiert sich in einer Vielzahl von Online-Foren, Facebook-Gruppen, Twitter-, Fernseh-, und 4Chan/8Chan-Beiträgen, Buchpublikationen und Bildern, die darauf abzielen, den politischen Raum entsprechend $\mathrm{zu}$ affizieren. In dieser gegen den Mainstream gerichteten Dynamik übernehmen rechte Populist*innen und Politiker*innen radikale Strategien, wie sie eigentlich aus den ästhetischen Avantgarden und der Kommunikationsguerilla der 1960er und 1970er Jahre bekannt sind: Skandalisierung, Störung, Provokation und Militanz. Nach dem Motto „Macht kaputt, was euch kaputt macht“ greift die Alt-Right Traditionen, Sitten und Gebräuche, Höflichkeiten und politische Konventionen an - immer unter der Maßgabe, dies sei ein legitimes Mittel im Kampf gegen die Meinungsdiktatur der liberalen Eliten. Angesichts einer Rhetorik des gesellschaftlichen Verfalls und der Sehnsucht nach Erneuerung überrascht es wenig, dass Trump die etablierten kulturellen und politischen Institutionen in ihrer aktuellen Verfassung für unrettbar erklärt. Hier spiegelt sich noch einmal der symbolische Überschuss des Correspondents' Dinner: Als Party, in der sich das Establishment selbst feiert, wird die jährliche Veranstaltung in Trumps Lesart zum Kristallisationspunkt der Washingtoner Dekadenz, die enden muss, damit es mit Amerikas Größe wieder bergauf gehen kann: „The Dinner is DEAD as we know it!“

Diese Perspektive ebnet den Weg für eine Analyse von symbolischen Tauschverhältnissen, für die die Persona Donald Trump einen ausgezeichneten Spürsinn entwickelt zu haben scheint. Trump startete in seinen Wahlkampf als Großprofiteur des kapitalistischen Systems. Für Medien und etablierte Politik war er zunächst kein ernstzunehmender Kandidat. Im Gegenteil: Trump wurde medial präsentiert als ein Schmuddelkind, im eigenen Verhalten von einer Politik der Herabsetzung bestimmt, gleichzeitig aber keineswegs so souverän, wie er selbst gerne von den anderen gesehen worden wäre. So wird er selbst zu einer Kippfigur, die wahlweise als Verlierer und/oder Gewinner in Erscheinung treten kann. Davon erzählen die beiden Teile seiner Autobiografie The Art of the Deal (1987) und The Art of the Comeback (1997). Sein augenscheinlicher politischer Dilettantismus transformiert sich, begünstigt von einem komplexen Ermöglichungszusammenhang, in 
den die medialen Verhältnisse ebenso hineinspielen wie eine grassierende Politikverdrossenheit, Zukunftsängste und eine erstarkende Alt-Right-Bewegung, in eine Darstellungs- und Aufmerksamkeitsressource. Je weniger Trump sich politischen Konventionen und den entsprechenden Sprachspielen unterwarf, je mehr er einen Mangel an politischem Können preisgab, desto mehr rückte er in den Fokus der etablierten Massenmedien, die sich von seiner Performance Spektakel und Polarisierung versprachen: Trump erhielt mehr mediale Aufmerksamkeit als alle anderen republikanischen Kandidaten, dreimal mehr Berichterstattungen als Hillary Clinton und 16 Mal mehr Sendezeit als Bernie Sanders (Pickard 2018: 195). Dies entspricht einer kostenlosen Werbung, die sich auf einen Wert von mehreren Milliarden Dollar beläuft. Je umfassender Medien und Politik sich darauf einschossen, in Trump nicht mehr als eine chancenlose, aber in ihrer Inkompetenz amüsante Randfigur zu sehen, desto mehr konnte sich der Millionär im öffentlichen Diskursraum als Underdog inszenieren, der seine Tatkraft schon auf dem Feld der Wirtschaft mit seiner Erfolgsgeschichte bewiesen hatte. In den Augen seiner Unterstützer*innen verkörpert er das Versprechen, die „gute alte Zeit“ des Wohlstands und der überschaubaren Verhältnisse wiederherzustellen. In Trumps Anti-Globalisierungs-Programm tritt vertikale an die Stelle von horizontaler Mobilität. Sein Angebot zielt auf die Schaffung exklusiver Solidarität und auf die Einrichtung einer Schutzzone, die getragen wird von der Idee (white) „America First“. Für ihre technische Umsetzung braucht es den Austritt aus internationalen Vertragsverhältnissen, einen Muslim Ban und den Bau einer Mauer.

Ein weiteres Spielfeld, das für das Theater der Souveränität von großer Relevanz ist, ergibt sich aus der Dynamisierung der Geschlechterordnung. Trump ist die Antwort auf die vermeintliche Krise weißer Männlichkeit, wie sie in Kreisen der Alt-Right impulsiv beklagt und symbolisch bearbeitet wird (Donovan 2012). Auch hier kann Trump seine vermeintliche Randständigkeit strategisch nutzen: Je weniger er modernen Männlichkeitsvorstellungen entspricht - insofern er den Logos bürgerlicher Rede unterläuft oder aus der repräsentativen Ästhetik des distinguierten Geschmacks herausfällt -, desto eher kann er die Hegemoniekritik feministischer Kämpfe zugunsten einer Zurschaustellung toxischer Maskulinität ignorieren. ${ }^{12}$ Seine Ausbrüche werden von verständnisvollen Wähler*innen als Ausdruck seines Nonkonformismus oder geschlechterstereotypischen Ungenügens gelesen und bieten etwa sexistischen Männern die Identifikationsfläche eines Leidensgenossen, der nun antritt, um mit den Zumutungen der Gleichberechtigung und Inklusion aufzuräumen (Dietze/Strick 2017). Trumps „Kunst des Erfolgs“

\footnotetext{
${ }^{12}$ Simon Strick entwickelt zum Thema unangemessener Männlichkeit den Begriff Inadequate Affect. Er beschreibt eine „kompensatorische Männlichkeitsformel“, die paradoxerweise durch die Überwindung der eigenen Inadäquatheit aufmerksamkeitsökonomisch attraktiv wird. Zwar können Figuren wie Trump Strick zufolge den Inadequate Affect in eine legitime Männlichkeit und Macht umwandeln und damit als Ressource der Restauration nutzen, simultan zeige diese „Brüchigkeit männlicher Herrschaft“ aber die Unangemessenheit Cis-männlicher Rollenbilder auf, womit sich ebenso eine Minorisierung (im Deleuzschen Sinne) von Cis-Männlichkeit vorantreiben ließe, vgl. Strick 2018.
} 
etabliert neue Äquivalenzketten, innerhalb derer Fakten und Fiktionen problemlos miteinander vertauscht werden können: An die Stelle der ritualisierten Pressekonferenz tritt der kommunikative Exzess; die Verpflichtung zur Konsensbildung wird abgelöst von der spektakelaffinen Schmährede; die differenzierte politische Debatte wird ersetzt durch den antagonistischen Tweet. Trump muss nicht mehr an Dinners teilnehmen, da er die Bühne im Hilton gegen das Everyday-(Social Media) Life eingetauscht hat und die Schmährede dort als souveränen Sprechakt nutzt. Es ist diese Kommunikationsform, die Teile der Öffentlichkeit dazu verleitet, seine politische Praxis als Satire, als parodistisches Element zu interpretieren. Trumps Souveränitätsinszenierung erreicht ein neues Niveau, weil er aufgrund der digitalen Medien nicht mehr auf die sozialen Nahräume des politischen Betriebs zur Verbreitung seiner politischen Initiativen angewiesen ist.

Stellt man die Analyse von Trumps Symbolpolitik in den Fragehorizont der politischen Ökonomie, drängt sich eine materialistische Perspektive auf. Trump steht für all jene Akteur*innen, die in einem kapitalistischen System stets nach oben fallen. Jeder sexistischen, rassistischen, xeno- oder homophoben Äußerung zum Trotz bleibt er für seine Wählerschaft eine attraktive Option. Gerade dann, wenn die Aufregung groß ist, wird er von seinen Wähler*innen als vertrauenswerter Führer rezipiert. Der Trumpismus funktioniert, weil er sein Publikum niederschwellig adressiert und eine gewinnbringende Teilnahme ohne ökonomischen Investitionszwang in Aussicht stellt: Die Möglichkeit, aus einem Mangel Kapital zu schlagen, die Inadäquatheit nicht zu verheimlichen, sondern im Gegenteil auszustellen und zu zelebrieren. Trump selbst macht es vor: Was zählt, ist die Fähigkeit, Ahnungslosigkeit als Unvoreingenommenheit zu verstehen und Inkompetenz selbstbewusst als frischen Blick zu verkaufen. Wer dazu in der Lage ist, sich von anderen Meinungen nicht irritieren oder beschämen zu lassen, kann es bis ganz nach oben schaffen. Nicht von ungefähr lautet Trumps Inklusionsversprechen in seiner Declaration Speech vom 16. Juni 2015: „Sadly, the American Dream is dead. But if I get elected president, I will bring it back bigger and better and stronger than ever before and we will make America great again" (Washington Post Staff 2015). So wird auch sein Fernbleiben vom Correspondents' Dinner 2018 durch Tweets gerahmt, die die zentrale Friktion des Wahlkampfs wiederholen: Abwesend in einer Arena des alten Establishments, ist er anwesend bei einer Veranstaltung in Michigan, an einem Ort also, der genau mit jenem Rust Belt des Mittleren Westens assoziiert wird, der aufgrund seiner siechenden Industrie ein Fallstrick für Hillary Clintons Präsidentschaftsambitionen war. Michelle Wolf fasste diese Hintergrundgeschichte des Dinners mit einem Scherz prägnant zusammen: ,It is kind of crazy that the Trump campaign was in contact with Russia, when the Hillary Campaign wasn't even in contact with Michigan“ (C-Span 2018).

Das Problem der Prekarisierung allein am Klassismus fest zu machen, würde allerdings genau jenen binären Logiken in die Hände spielen, die das vorgestellte Tauschprinzip einführt. Trumps Angebot einer neuen Solidarität ist exklusiv. Es richtet sich an eine Klientel von zu Opfern stilisierten weißen Männern und schließt andere interdependente soziale Gruppen (Latin*x, Frauen, Schwarze usw.) programmatisch aus. Während die Abstiegs- und Entsicherungsängste 
der weißen Mittelschicht neue Anerkennung erfahren, spricht der Trumpismus Anerkennungsforderungen anderer marginalisierter Gruppen jede Berechtigung ab. Sie fallen nicht nur aus dem Aufmerksamkeitsfokus heraus, sondern werden von Trump indirekt als Symptome des gesellschaftlichen Verfalls markiert - zu Hassobjekten gemacht. Auch hier ist eine doppelte invektive Dynamik am Werk: So fordert die Alt-Right eine Zurückdrängung der identitätspolitischen Gleichstellungsforderungen bislang marginalisierter Gruppen im Namen der Meinungsfreiheit. Der Verweis auf das Free Speech-Recht, wie es von Kritiker*innen einer diskriminierungssensiblen Sprache immer wieder vorgebracht wird, fungiert de facto als die Einforderung eines Rechts auf Hate Speech. Gleichwohl ist der Bezug auf Free-Speech strategisch erfolgreich, schafft er es doch, bestimmte Diskurszonen in den Fokus zu rücken und andere Bereiche abzuschatten. In der öffentlichen Darstellung der wirtschaftlichen und kulturellen Abstiegsängste der weißen Mittelschicht bleiben existenzielle Nöte marginalisierter Bevölkerungsgruppen, die um ihre sexuelle Integrität oder ihre physische Sicherheit fürchten müssen, daher zunehmend ausgeblendet. Umso bedenklicher ist es, dass sich die Akteur*innen des erstarkenden Populismus in den USA und in Europa in Hasstiraden gegenüber Political Correctness (PC) ergehen, in denen sie voller Stolz ihre -ismen (Rassismus, Sexismus usw.) im Namen von gutem ,Volksempfinden', gesundem Menschenverstand und natürlicher Ordnung ausleben. Machtsensible Aufforderungen wie „Check your privilege“ werden als Totschlagargumente privilegierter „PC Principles“ (South Park 2015) zurückgewiesen, da diese andernfalls in doppelter Hinsicht eine Irritation erzeugen würden: Zum einen in der Perspektive ,nach unten', insofern die eigenen, nicht alleine über Leistung vermittelten finanziellen Vorteile und Privilegien in der Gesundheitsversorgung, im Bildungsbereich usw. eingestanden werden müssten. Zum anderen in der Orientierung ,nach oben', weil ein Vergleich auch hier ergeben würde, dass es gläserne Decken gibt, die dem eigenen Aufstiegsstreben finanzielle und symbolische Grenzen setzen. Laut Lauren Berlant reagiert das emotionale Angebot des Trumpismus auf die Wahrnehmung, sich im herrschenden Diskurs moralisch diskreditiert und bevormundet zu finden. Trump verkörpere dagegen das Versprechen einer neuen Freiheit: „Trump is free. [...] Trump people don't use suffering as a metric of virtue. They want fairness of a sort, but mainly they seek freedom from shame" (Berlant 2016). Sich mit der Einsicht konfrontiert zu finden, zugleich mit zu vielen und zu wenigen Privilegien ausgestattet zu sein, begründet eine soziale Positionierung, von der aus ganz unterschiedliche Akteur*innen einen Status der Minorisierung für sich beanspruchen. Erfolg versprechend sind solche Forderungen dann - dies haben die identitätspolitischen Kämpfe der letzten Jahre und Trumps Weg zur Präsidentschaft vorgeführt -, wenn sie es verstehen, das Moment der Scham als am eigenen Leib erfahrene Kränkungsgeschichte diskursiv produktiv zu machen. Die Selbstakklamation als Opfer einer Beschämung - durch die Medien, die Politik, den Staat - kann eine so starke Evidenz entwickeln, dass diese die Erklärungsversuche der gegenwärtigen Konjunktur des Populismus dominiert. So kommt es, dass diverse Stimmen die Sorgen dieser Bürger*innen zu einseitig als Ergebnis identitätspolitischer Versäumnisse, der neoliberalistischen Kränkung oder der 
Marginalisierung der weißen unteren Mittelschicht herleiten (Eribon 2016; Lilla 2017; Kreiss 2018). In dieser Diskurslogik ist es wenig überraschend, dass das Correspondents' Dinner 2011 als eine traumatische Geburtsstunde Donald Trumps und als ein Wechsel der Minderheitenposition nach dem Motto der gleichnamigen TV-Serie Orange is the New Black (Netflix seit 2013) interpretiert wurde. Dieser Abend, an dem der Schwarze Präsident den weißen Konkurrenten vor den Augen der Nation zur Lachnummer machte, steht symbolisch für die hier beschriebenen Mechanismen der Macht- und Schamumkehrung, bzw. -aneignung.

$\mathrm{Zu}$ Trumps Politik der Herabsetzung gehört, dass dieser in der Lage ist, beide Positionen des Invektivierungsspiels zu besetzen. Trumps Diskursstrategie changiert zwischen der Rolle des Opfers und der des dominanten Souveräns. Entscheidend für Trumps Affizierungs- und Mobilisierungsstrategie ist es, dass er seine Angriffe auf politische Konkurrent*innen und Kritiker*innen zumeist als eine Art Vorwärtsverteidigung zu rahmen weiß. Diese Fähigkeit des Präsidenten, situativ zwischen unterschiedlichen Sprecherpositionen zu wechseln, resultiert aus dem Umstand, dass Trumps öffentliche Rede nicht sachlich orientiert, sondern primär moralisch codiert ist. Gerade in der Auseinandersetzung mit verhassten Nachrichtensendern - dies war das Thema des Komikers Hasan Minhaj beim Correspondents' Dinner 2017 - nutzt Trump jeden Fehler und jede Ungenauigkeit in der Berichterstattung, um entrüstet gegen den vermeintlichen Mainstream Stimmung zu machen (ABC News 2017). Angesichts einer von Trump geschürten Krise des Journalismus, der seine Akzeptanz und Autorität als Agentur öffentlicher Meinungsbildung bei Teilen der US-amerikanischen Bevölkerung weitgehend eingebüßt zu haben scheint, erinnert Minhaj die anwesenden Journalist*innen daran, dass sie sich keine Fehler mehr leisten könnten. Anstelle Trump weiter mit journalistischer Arroganz zu begegnen, sei es geboten, mit sachlicher Präzision und ohne denunziatorische Aufregung das Vertrauen ihres Publikums zurück zu gewinnen:

\footnotetext{
„I don't have a solution on winning back trust. I don't. But I know, in the age of Trump, I know, that you guys have to be more perfect now, more than ever. Because you are how the president gets his news. Not from advisors, not from experts, not from intelligence agencies. You guys. You got to be on your A-Game, you got to be twice as good. You can't make any mistakes. Cause if one of you messes up, he blames your entire group.... - and now you know what it feels like to be a minority! [...] I've got three decades of experience being brown. So, if you want to survive the age of Trump you've got to think like a minority. [...] By the way, you guys aren't really minorities. You guys are super white. I can see MSNBC going ,We've got our minority card!" - no“" (ABC News 2017).
}

Was Minhaj hier anmahnt, ist nichts anderes als eine Anpassung des journalistischen Selbstverständnisses an ein sich wandelndes Spielfeld. Unter den Bedingungen sozialer Medien und eines sich zusehends hybridisierenden Mediensystems ist nicht mehr ausgemacht, dass die journalistische Berichterstattung in ihrer Kontroll- und Kritikfunktion politischer Macht auf demokratische Akzeptanz stößt. Ein Blick auf die neuen populistischen Bewegungen weltweit zeigt, dass die Vierte Gewalt in der Kritik steht. Diese Einschätzung trifft auf Trump und seine Agitation gegen die Fake News ebenso zu wie auf die Medienschelte 
eines Victor Orbán, eines Matteo Salvini oder eines Björn Höcke. Die Logik einer symbolischen Selbstminorisierung ist immer die gleiche: Journalist*innen werden zu Agent*innen eines nebulösen, mächtigen Systems stilisiert, das über ihre Medienkanäle das Volk manipuliere. Dagegen gelte es Widerstand zu organisieren. Die zugrundeliegende Affektdynamik ist alles andere als neu: Die Geschichte der Moderne ist voller Phantasmen jüdischer und kommunistischer Weltverschwörungen. Der jeweilige Führerkult geht dabei immer mit einem ,Opferkult' einher, der Appell an ein handlungsbereites ,Wir' wird stets getragen von einer politisch einflussreichen Verschwörungstheorie, die ihre Evidenz aus der Behauptung folgenschwerer Fake News ableitet. Neu an der aktuellen Lage sind die Resonanzstärke und der massive Umbau der Zeigbarkeits- und Sagbarkeitsordnung. Eine Entwicklung, die so Anfang des Jahrtausends noch nicht in diesem Ausmaß vorstellbar gewesen wäre. Begünstigt von der many-to-many-Kommunikation der digitalen Medien, deren Tendenz zur Fragmentierung der Öffentlichkeit und zum Aufbau sich einander misstrauisch bis feindlich gegenüberstehender „Neogemeinschaften“ (Reckwitz 2017: $261 \mathrm{ff}$.), hat der Journalismus die Rolle als Gatekeeper öffentlicher Diskursbeteiligung verloren. Die durch soziale Netzwerke und alternative Medien erzeugte Zentrifugalwirkung hat zur Folge, dass der Glaube an den zwanglosen Zwang des besseren Arguments, der noch nie wirklich den Realitätstest ausgehalten hat, heute völlig in die Irre läuft. Faktenchecks und differenzierte Problemanalysen überwinden kaum noch die Irritationsschranken von Filterblasen, Echokammern und Verschwörungsnarrativen. Gleichzeitig - und das wäre einer vorschnellen dystopischen Kulturkritik der Medien zu entgegnen begünstigen digitale Technologien zugleich eine wichtige Pluralisierung von Wissensbeständen und bieten Kommunikationskanäle für politische Auseinandersetzungen, die die alten Gatekeeper unzureichend abdeckten. Zeynep Tufekci warnt in diesem Sinne zu Recht vor zu einseitigen Analysen von medialen Aushandlungsprozessen, die dazu tendieren, eine gute alte Zeit zu idealisieren: Zwar haben die traditionellen Gatekeeper wichtige Beiträge zur Qualitätssicherung der Nachrichtenberichterstattung geliefert. Gleichzeitig haben sie aber durch ihre oftmals selbst wiederum nicht reflektierten Schwerpunktsetzungen auch blinde Flecken und tote Winkel der öffentlichen Aufmerksamkeit erzeugt. Gerade die oft zu unkritische Berichterstattung habe demnach im Verbund mit der Gleichgültigkeit der Eliten zu einer politischen Kultur geführt, die anfällig sei für patriotisches Trolling (Tufekci 2018). Bezogen auf die Entwicklung populistischer Echokammern schreibt sie: „Our cognitive universe isn't an echo chamber, but our social one is“ (ebd.). In der Konsequenz dieser Konstellation treten Fakten zugunsten von Zugehörigkeiten in den Hintergrund.

Trumps Nähe zu Russland zu untersuchen oder jede einzelne seiner zahlreichen Lügen und Halbwahrheiten mit investigativer Energie auf politifact. com aufzudecken, ist jedenfalls ebenso mühselig wie in seinen Effekten ambivalent. Im Sinne einer demokratischen Kritik möglichen Fehlverhaltens bleiben direkte juristische Konsequenzen aus. Wohl aber ist die Folge eine weitere Verhärtung der einander gegenüberstehenden politischen Lager, die immer weniger von einem Grundkonsens politischer Zusammenarbeit bestimmt zu seien 
scheinen. Die Wirkungslosigkeit sachlicher Kritik resultiert aus einem narrativen Schutzmechanismus, der wiederum viel mit der affektiven Konstellation von Herabsetzung und Beschämung zu tun hat. Im Englischen verweist das Verb „to expose“ auf zwei unterschiedliche Bedeutungszusammenhänge. Einerseits kann „expose“ „Aufdeckung, Freilegung“ bedeuten. In diesem Sinne ist „exposure" das Ziel investigativer journalistischer Arbeit. Zum anderen kann die Formel „to expose something/someone“ aber auch auf eine Praxis der Bloßstellung verweisen. Beide Bedeutungen überblenden sich im Verhältnis der Medien zu Trump und fallen zugleich in der politischen Prädisposition unterschiedlicher Nachrichtenrezipient*innen wieder auseinander. Diejenigen, die Trump nicht gewählt haben, werden von jeder neuen Information in ihrer Ablehnung bestätigt. Die Trump-Unterstützer*innen aber sehen in der journalistischen Auseinandersetzung mit ihm den Versuch der Bloßstellung. Aus Informationen werden im Abdichtungsnarrativ schließlich Elemente einer perfiden Diffamierungskampagne, mit deren faktischem Gehalt man sich erst gar nicht beschäftigt. Es gehört zur Logik der Epistemologie des Closet (Sedgwick 1990), dass ein fremdbestimmtes Outing als Beschämungsversuch interpretiert werden kann. Jede ans Licht gebrachte „Perversität“ birgt sogleich die Chance, in einem Akt der Selbstaffirmation zu einem Nachweis des Tugendterrors des Establishments umgemünzt zu werden. Im Falle von Trumps Aneignungsstrategie kann in einer Situation starker gesellschaftlicher Polarisierung mit einer entsprechend resonanzstarken Anschlusskommunikation gerechnet werden. Jegliche Aufdeckung wird dann zur identitätspolitisch funktionalisierbaren Bloßstellung, mit der die Kritiker*innen zur Schließung der eigenen Reihen motiviert werden. Minhajs Rat ,if you want to survive the age of Trump you've got to think like a minority“, scheint vor allem Trump selbst zu befolgen.

\section{Orange is the New Black Box}

Sieht man in Trump einen Parasiten des Medien- und Empörungsspektakels, der die Entrüstung der Anderen als Werkstoff der eigenen Identitätspolitik missbraucht, so hat das Konsequenzen für den eigenen kommunikativen Umgang mit dem US-Präsidenten. Anstatt immer weiter im Täter-/Opfer-Zirkus mitzuspielen und neues Futter für Trumps Tweets und Rants bereitzustellen, gilt es, dieses ökonomische Prinzip der wechselseitigen Gabe (Mauss 1968) zu durchbrechen. Dazu gehört allerdings auch, sich nicht an der protoapokalyptischen Empörungswelle der nach wie vor machtvollen und stabil institutionalisierten Nachrichtenmedien zu beteiligen, die Trumps Behauptungen dankbar aufgreifen und durch alarmistische Berichterstattung in profitable Aufmerksamkeit verwandeln. Wer sich, ökonomischen Kalkülen folgend, in einem Akt der Selbstviktimisierung die Position des Minoritären zuschreibt, hat bereits die Spielregeln von Trumps Politik der Herabsetzung akzeptiert. Michelle Wolfs Witz „Trump is so Broke! How broke is he? He grabs pussies because he thinks there might be loose change in them!“, spiegelt die derzeitige politische Realität wider. Sexismus offen auszustellen ist 
nicht mehr hinderlich für den politischen Werdegang, sondern profitabel für das eigene Status-Management. Weil dem Vorwurf von Sexismus erfolgreich mit dem Verweis auf eine versuchte Bevormundung begegnet werden kann, ist es mittlerweile wieder möglich geworden, dass Frauen innerhalb der politischen Arena für ihr Aussehen attackiert werden. Auch sind Berichte von sexuellen Übergriffen bei der Aufnahme in den Supreme Court kein unüberwindbares Problem mehr, zumindest solange nicht, wie es gelingt, Zweifel an ihrem Wahrheitsgehalt in relevanten Teilen der Öffentlichkeit virulent zu halten. Trumps Misogynie, aufgezeichnet in einem Video, das seine Prahlerei mit einer sexuellen Belästigung dokumentiert, hat Schule gemacht: Dort, wo die Hegemonie eines virilen Männlichkeitsbildes in Gefahr ist, reicht es aus, die Integrität der Opfer so lange medial infrage zu stellen, bis die Tat schlussendlich als ein Moment impulsiver Virilität erscheint.

Dies unterscheidet Trump, zumindest der Intensität nach, von anderen rechtspopulistischen Störfiguren seiner Zeit. Er ist ein Präsident, der ohne jede Integrität auskommt. In seinem Handeln stets an Anschlusskommunikation und Verwertung interessiert, agiert Trump als diffuses Anpassungsgefüge, das situativ Dinge verwerfen und wiederaufnehmen kann. Gerade durch diese Wechselverhältnisse und die oben beschriebenen Angriffe von außen gewinnt er an Kontur und Substanz. Aufgrund seiner eigenen Adaptionsfähigkeit und der Aufmerksamkeitsstruktur seiner Wählerschaft ist es Trump möglich, selbst Wolfs Komikeinlage produktiv misszuverstehen und ihre auf Sexismus anspielenden Scherze über die Pressesprecherin Huckabee Sanders als PR-Material für seine parallel stattfindende Veranstaltung in Michigan zu nutzen. Hierbei wird nicht nur auf einer allgemeinen Ebene Klassismus und Sexismus gegeneinander ausgespielt, sondern zugleich auch der eigene Sexismus verneinend bearbeitet. Donald Trump ist so betrachtet nicht nur ein Great Disruptor, der Störungen innerhalb medialer und politischer Ordnungsgefügen produziert. Er ist zugleich auch ein Great Adaptor, der eine kontinuierliche Mimikry und Mimese seiner medialen Umwelt betreibt. Indem er in seiner Kommunikation eine Transponierung und Invertierung kritischer Inhalte leistet, schafft er es, neue Rahmen der Beobachtung und Rezeption seiner Äußerungen herzustellen. Durch diese Genreverschiebungen vermag Trump zu irritieren und genau jenes Begehren bei seinen Kritiker*innen zu triggern, die ihn in der Arena der Wahrheit stellen und zur Verantwortung ziehen wollen. Die manische Dauerbeschäftigung mit Trumps Psyche ist eine der Reaktionen auf die Unmöglichkeit, Erwartbarkeit als Grundlage politischer Prognose herzustellen. Fragen wie ,What is he thinking?‘, ,Is he a narcissist because of his father?`, ,Did he conspire with the Russians?‘, ,Was it his idea or rather Roger Stones?` oder ,Does he really intend to start a nuclear war because of his ego?' sind Ausdruck des Unvermögens, sich von der alten Vorstellung der Sachgebundenheit politischer Strategie- und Entscheidungsfindung zu lösen. An Trumps numerischen Populismus jedoch, für den alleine Klickzahlen, Likes und Retweets Indikatoren des Erfolgs sind, scheitern solche Zuschreibungen. Trump hat eher Ähnlichkeit mit einem Chat-Bot, einem selbstlernenden Algorithmus mit Social Media Account. Man erinnere sich etwa an die von Microsoft entwickelte künstliche Intelligenz „Tay“, 
die mit eigenem Twitter-Account - @ TayandYou - ausgestattet und darauf programmiert war, die Sprache von Twitter-Nutzer*innen zu imitieren (Dewey 2016; Bright 2016). Microsofts Slogan zu dem Projekt lautete: „The more you chat with Tay, the smarter she gets" (Hunt 2016). Der selbstlernende Bot entwickelte sich allerdings innerhalb weniger Tage zu einem Image-Debakel: Aufgrund seiner das Verhalten von Twitter-Nutzer*innen imitierenden antisemitischen, sexistischen und rassistischen Aussagen wurde Tay zum Paradebeispiel für AS (,Artificial Stupidity“, Ferguson 2016). Tay wurde daraufhin nach nur wenigen Tagen vom Netz genommen - nachdem sie mitunter bereits Trump-Supporterin geworden war (,Trump is the only hope we've got“, Hunt 2016).

Es erscheint daher zielführend, Trump vor allem hinsichtlich seines transversalen Bewegungsvektors zu beobachten, der ihn quer zu den gesellschaftlich etablierten Ordnungen des Sagbaren agieren lässt. Eine solche Perspektive fragt zuerst nach der Funktionalität seiner Kommunikationsweise, ohne die Analyse zugunsten einer moralischen oder psychologischen Wertung vorschnell abzubrechen. Als Anpassungs- und Aneignungsgefüge zieht Trump in immer neue Richtungen und lässt sich von ihnen ziehen und damit auch sprichwörtlich „gehen“. Statt sich immer wieder an der Frage nach Trumps „Sein“ abzuarbeiten, ist es produktiver, den US-Präsidenten als eine Black Box zu konzeptualisieren, die mit ihrer jeweiligen Umwelt interagiert und deren Outputs von den vorausgegangenen Inputs abhängig sind.

Kybernetik und Systemtheorie verstehen unter Black Box ein komplexes System, dessen innere Struktur für Analysen nicht zugänglich ist. Daher verschiebt sich das Interesse vom Wesen der Black Box hin zur Beschreibung ihrer In- und Output-Beziehungen (Luhmann 2006: 153 ff.; Latour 2002: 373). Bruno Latour weist in seinen Untersuchungen zur Technikgeschichte darauf hin, dass blackboxing als Prozess zu verstehen sei, der sich aus der Erfolgsgeschichte wissenschaftlicher oder technischer Artefakte oder Praktiken ergibt: Wenn Maschinen effizient sind, wird im alltäglichen Umgang mit ihnen die interne Komplexität immer unwichtiger - so lange etwa der Computer funktioniert, brauchen sich Verbraucher*innen nicht um seinen inneren Aufbau zu kümmern. Latour geht von der These aus, dass sich paradoxerweise die Opazität eines Artefakts erhöht, wenn es funktional erfolgreich ist. Nicht nur für die Analyse der Politik könnte eine methodische Inspiration der Actor Network Theory lauten, nicht mehr primär nach den Intentionen der beteiligten Akteur*innen zu fragen, sondern die jeweils gegebenen diskursiven Ermöglichungszusammenhänge und medialen Netzwerke in den Mittelpunkt zu stellen und darauf zu achten, welche In- und Outputs situativ miteinander korreliert sind. Anstatt in der Tradition journalistischer Investigation die Black Box Trump öffnen und ihren Inhalt exponieren zu wollen (Woodward 2018), erscheint es lohnender, die Aufmerksamkeit auf die jeweiligen Milieus und die ihnen zugrundeliegenden Machtkomplexe zu richten. Dazu gehört es, unter diskursfunktionalen Gesichtspunkten die kontinuierlich von Trump prozessierten gesellschaftlichen In- und Outputs zu befragen, aber auch nach jenen Sozietäten und politischen Formen zu fahnden, die bisher nicht einheg- oder adaptierbar sind und die Black Box Trump in ihrer Funktionalität unterbrechen könnten. Neben 
einer Untersuchung der jeweiligen Ökologien, die etwa eine Präsidentschaft Trumps erst realisierbar machen, gilt es ebenso jene sozialen und ästhetischen Existenzweisen (Maihofer 1995) in die Aufmerksamkeitsökonomie einzubinden, die sich solchen politischen Figuren wie Trump stets entziehen. Dazu gehören etwa die antirassistischen Bewegungen und Paraden in (US-)amerikanischen und europäischen Städten, die Solidarity/Sanctuary Cities-Initiativen, die Women's Marches. Ein Blick auf In- und Outputs ermöglicht es - um die Metaphorik fortzuführen - Donald Trump als Verstärker gesamtgesellschaftlicher Diskurse und Affektdynamiken zu betrachten, aber gleichzeitig auch als einen Prozessor, der „eingreifende Veränderungen“ (Winkler 2015: 29) in einem kontinuierlichen Fluss an Daten vornimmt, Außenraum mit Innenraum verbindet bzw. re-arrangiert und damit als Subjekt „selbst zum Relais des medialen Dispositivs“ (ebd.: 109) wird.

Unter Rückgriff auf die Apparatus-Theorie, die den Kinoraum als Black Box konzeptualisiert, lädt noch ein weiterer Aspekt an der vorgeschlagenen Perspektivierung von Trump zum Weiterdenken ein: Die Black Box, so Hito Steyerl, kann im Unterschied zum White Cube des Ausstellungsraums ,als Wunschmaschine gedacht [werden], die von unbewusstem Begehren, Trieben und libidinösen Identifikationen durchdrungen ist" (Steyerl 2017: 138). Ihre schwarze Farbe ist assoziiert mit Vorstellungen von „Sinnlichkeit, Affektivität, Magie und Irrationalität“ (139). Die Black Box ist ,in unbewusste und unkontrollierbare Dynamiken verwickelt" (ebd.), sie manipuliert Affekte. Die Farbcodierung hat in Steyerls Beschreibung eine analytische Dimension: Die Black Box webt sich hier in eine rassisierte hell/dunkel-Metaphorik ein (ebd.). Sie umreißt begrifflich einen affektiven Raum, der im Dunkeln verbleibt und der Vernunft nur unzureichend zugänglich ist. Steyerl arbeitet heraus, dass die Black Box als Ort der Illusion, der Verführung, des Spektakels aber auch als ein Ort der Überwältigung und des unterdrückten Unbewussten diskursiviert wird. Die Black Box markiert das absolute Gegenteil der aufgeklärten Welt des White Cubes, der mit dem distanzierten Blick eines autonomen und kontemplativen Subjekts verbunden ist, das souverän den Objekten im Museumsraum gegenübertritt. Die Black Box wird in einer kontradiktorischen Gegenüberstellung zum „Erscheinungsraum einer zugleich profanen und illusorischen Wirklichkeit" (ebd.). Die Filme, die in den dunklen Räumen des Kinos oder des Museums gezeigt werden, realisieren eine eigene Zeitökonomie und prägen den Betrachter*innen ein Blickarrangement auf. Indem die Black Box eine eigene perzeptive Agency entwickelt, der sich die betrachtenden Subjekte unterwerfen müssen, erzeugt sie eine mit der Gefahr des Souveränitätsverlusts einhergehende Angstlust (Groys 2001; Steyerl 2017: 140). Black Box und White Cube stehen in einem wechselseitigen Konflikt, der von einem Bedrohungsszenario befeuert wird, in dem „Licht und Dunkel, Souveränität und Verunsicherung, Essenz und Schein“ in einem vermeintlich unüberwindbar binären Modell aufeinandertreffen (ebd.: 141). Anhand dieses kleinen Exkurses in die Medientheorie wird noch einmal von anderer Warte aus nachvollziehbar, welche affektive Dynamik das Verhältnis der Nachrichtenmedien zu Trump organisiert: Eine von Angstlust getriebene Beobachtungsmanie nämlich, die in Trump ein Anderes jeglicher politischer Rationalität zu erkennen 
vermeint, von dem sie sich aufgrund ungläubiger Faszination und profitorientierter Skandalisierungserwartung nicht lösen kann. Gerade weil Trump als disruptive Anomalie narrativiert werden konnte, unterwarfen sich die Medien bereitwillig seinen Aufmerksamkeits- und Zeitökonomien - Trump Tower, Trump Twitter, Trump Time usw. In der Retrospektive erscheint es mit Blick auf die Anfangszeit der politischen Karriere Trumps ebenso nachvollziehbar wie ironisch, dass die traditionellen Nachrichtenmedien fortwährend versuchten, die Black Box Trump in den White Cube zu überführen und dabei genau jene Bedrohungsphantasmen und Herabsetzungen zwischen Black und White reproduzierten, die sie an Trumps Populismus kritisierten. Die Hypostasierung Trumps zum irrationalen, eitlen Sittenmonster (Foucault 2007) verbleibt genau in jenen auf Binarismen gebauten Tauschverhältnissen, aus denen Trump das Charisma und die Authentizitätseffekte erwirtschaften kann, die ihn für zahlreiche Wähler*innen als vermeintliche Systemalternative erst attraktiv machen.

Die Untersuchung der medialen, politischen und diskursiven Ökologie Trumps erlaubt es schlussendlich auch, den Eintritt in ein weiteres problematisches Tauschverhältnis zu vermeiden, das sich anhand von Michelle Obamas Ausspruch „When they go low, we go high“ beim Democratic National Convention 2016 konturieren lässt (Washington Post Staff 2016). Das Zitat bildete die Quintessenz einer scharfen Abrechnung mit Trumps sexuell übergriffiger Vergangenheit und seinem fortdauernden und ostentativ zur Schau gestellten Sexismus. So wichtig Michelle Obamas feministische Positionierung und ihr Eintreten für eine gesamtgesellschaftliche Verantwortung und eine Praxis der Care-Work ist, befördert sie doch zugleich auch die Gefahr, dass ihr Auftritt von jenen Akteur*innen affektund diskurspolitisch ausgebeutet wird, denen sie eigentlich den öffentlichen Raum streitig machen will. Gemeint ist ein Mechanismus, den man als regulative Normalisierung bezeichnen kann: Je herabsetzender, beschämender, ungehaltener und deplatzierter sich politische Figuren wie Trump benehmen, desto eher wirken geringfügig moderatere Erscheinungs- und Handlungsweisen tolerier- oder gar akzeptierbar. Eine maßstabs- und geschichtsvergessene Kritik, die Trump zum außerordentlichen Great Disruptor erklärt und ihn jenseits der politischen Kultur der USA situiert, entzieht der Kritik selbst den Boden. Je eklatanter sich Trump islamophob, rassistisch und xenophob äußert und je mehr politische Energie er in den Bau einer Mauer legt, desto mehr droht in Vergessenheit zu geraten, dass es unter Barack Obama so viele Abschiebungen gab wie bei keinem anderen Präsidenten. Eine problematische Diskursrelation ist zu entdecken: Hillary Clintons feministische Positionierung angesichts von Trumps Sexismus entzieht ihre antimuslimische Außenpolitik der Diskussion; Horst Seehofers Behauptung, die Flüchtlingspolitik im Sommer 2015 sei die „Mutter aller Probleme“ gewesen, lässt die alles andere als unproblematische Abschiebungs- und Externalisierungspolitik Angela Merkels als human erscheinen. „When they go low, we go high“ verweist in diesem Sinne auf eine schleichende Rehabilitierung eigentlich kritikwürdiger Praxen und auf nachhaltige Diskursverschiebungen rechtspopulistischen Gedankenguts in das Zentrum demokratischer Meinungsvielfalt. Umwelten reagieren auf Akteur*innen wie Trump, Le Pen oder Gauland. 
Es ist an der Zeit, die skizzierten Tauschmechanismen in ihren vielgestaltigen Ausprägungen zu rekonstruieren und in einem zweiten Schritt die Übertragungskanäle, die den Great Disruptor und seine politischen Adept*innen mit Energie versorgen, ihrerseits zu unterbrechen. Dafür ist es zunächst nötig, die kommunikativen In- und Output-Bilanzen des Rechtspopulismus in ihrer Funktionalität zu vergegenwärtigen und dabei die Rolle von Invektivität als Ressource und Dynamisierungsmoment politischer und sozialer Positionierungsprozesse genauer $\mathrm{zu}$ fassen. Erst wenn die parasitäre Logik der Politik der Herabsetzung genau erfasst ist, sind die Voraussetzungen zur Entwicklung einer nachhaltigen Gegenstrategie geschaffen. Deren Einsatzpunkt müsste es sein, die durch den neu-rechten Populismus forcierte Polarisierung des politischen Beschreibungsvokabulars, die identitäre Einteilungslogik von Freund und Feind, Wir und Sie, Oben und Unten, Innen und Außen usw. in ihren reduktionistischen Voraussetzungen und prekären affektpolitischen Effekten zurückzuweisen. Dazu gehört es auch, jene Bewegungen $\mathrm{zu}$ unterstützen, die sich mit ihrer politischen Praxis oder ihren Existenzbedingungen gegen rassistische Grenz- und Affektpolitiken, sexistische Gewalt, politische Schmähreden usw. stellen, statt sie zu ignorieren, zu verharmlosen oder gar zu teilen. Es ist möglich, Output und Reichweite der Black Box zu beeinflussen: durch Beschneidung der kommunikativen Ressourcen, die wir alle einer Politik der Herabsetzung zur Verfügung stellen.

\section{Literatur}

ABC News: Hasan Minhaj full White House Correspondents Dinner speech (29.04.2017), https://www.youtube.com/watch?v=uDmSZX_zVuQ (11.03.2019).

Aristoteles: Poetik. Berlin 2008.

Berlant, Lauren: Trump, or Political Emotions. In: The New Inquiry (05.09.2016), https://thenewinquiry.com/trump-or-political-emotions/ (13.03.2019).

Chadwick, Andrew: The Hybrid Media System. Politics and Power. Oxford 2013.

C-Span: President Obama at the 2011 White House Correpondents' Dinner (30.04.2011), https:// www.youtube.com/watch?v=n9mzJhvC-8E (11.03.2019).

C-Span: Michelle Wolf COMPLETE REMARKS at 2018 White House Correspondents' Dinner, (28.04.2018), https://www.youtube.com/watch?v=DDbx1uArVOM (11.03.2019).

Bright, Peter: Tay, the neo-Nazi millennial chatbot, gets autopsied. Microsoft apologizes for her behavior and talks about what went wrong. In: Arstechnica (26.03.2016), https://arstechnica. com/information-technology/2016/03/tay-the-neo-nazi-millennial-chatbot-gets-autopsied/ (13.03.2019).

Deleuze, Gilles/Guattari, Félix: Tausend Plateaus. Kapitalismus und Schizophrenie. Berlin 1993.

Dewey, Caitlin: Meet Tay, the creepy-realistic robot who talks just like a teen. In: The Washington Post (23.03.2016), https://www.washingtonpost.com/news/the-intersect/wp/2016/03/23/ meet-tay-the-creepy-realistic-robot-who-talks-just-like-a-teen/?utm_term $=.628 \mathrm{f} 23355458$ (13.03.2019).

Dietze, Gabriele/Strick, Simon: Der Aufstand der Betamännchen. In: Gender-Blog der Zeitschrift für Medienwissenschaft (18.12.2017), https://www.zfmedienwissenschaft.de/online/blog/ der-aufstand-der-betam\%C3\%A4nnchen (22.10.2018).

Donovan, Jack: The Way of Men. New York 2012.

Eisenhauer, Robert: Archeologies of the Invective. New York u. a. 2007.

Eribon, Didier: Rückkehr nach Reims. Berlin 2016. 
Fanon, Frantz: Die Verdammten dieser Erde. Frankfurt a. M. 1981.

Ferguson, Niall: Tay, Trump, and the artificial stupidity. In: The Boston Globe (04.04.2016), https://www.bostonglobe.com/opinion/2016/04/03/tay-trump-and-artificial-stupidity/9Zk3dP7CSByV5bzbc3L2vJ/story.html (13.03.2019).

Foucault, Michel: Die Anormalen. Vorlesungen am Collège de France 1974/1975. Frankfurt a. M. 2007.

Frevert, Ute: Die Politik der Demütigung. Schauplätze von Macht und Ohnmacht. Frankfurt a. M. 2017.

Garfinkel, Harold: Conditions of successful degradation ceremonies. In: American Journal of Sociology 61/5 (1956): 420-424.

Gehring, Petra: Über die Körperkraft von Sprache. In: Steffen K. Herrmann/Sybille Krämer/ Hannes Kuch (Hg.): Verletzende Worte. Die Grammatik sprachlicher Missachtung. Bielefeld 2007: 211-228.

Gopnik, Adam: Trump and Obama: A night to remember. In: The New Yorker (12.09.2015), https://www.newyorker.com/news/daily-comment/trump-and-obama-a-night-to-remember (14.03.2019).

Groys, Boris: On the aesthetics of video installations. In: Stan Douglas: Le Detroit. Basel 2001.

Grynbaum, Michael M.: No more laughs as White House Correspondents' Dinner turns to a historian. In: The New York Times (19.11.2018), https://www.nytimes.com/2018/11/19/business/ media/ron-chernow-white-house-correspondents-dinner.html (16.04.2019).

Grynbaum, Michael M./Rogers, Katie: New Yorker and Vanity Fair pull out of Correspondents' Dinner parties. In: The New York Times (03.02.2017), https://www.nytimes.com/2017/02/03/ us/politics/trump-white-house-correspondents-dinner.html (18.03.2019).

Haberman, Maggie/Burns, Alexander: Donald Trump's presidential run began in an effort to gain stature. In: The New York Times (12.03.2016), https://www.nytimes.com/2016/03/13/us/politics/donald-trump-campaign.html?_r=0 (13.03.2019).

Heil, Emily/Fahri, Paul: The White House correspondents' dinner breaks decades-old tradition by not featuring a comedian. In: The Washington Post (19.11.2018), https://www.washingtonpost.com/arts-entertainment/2018/11/19/white-house-correspondents-dinner-will-not-feature-comedian-first-time-decades/?utm_term=.d6bb1fbf1a7f (16.04.2019).

Hughes, Geoffrey: Political Correctness. A History of Semantics and Culture. London 2009.

Hunt, Elle: Tay, Microsoft's AI chatbot, gets a crash course in racism from Twitter. In: The Guardian (24.03.2016), https://www.theguardian.com/technology/2016/mar/24/tay-microsofts-aichatbot-gets-a-crash-course-in-racism-from-twitter (23.10.2018).

Janker, Karin: Journalisten mit Politikern auf Du und Du. In: Süddeutsche Zeitung (26.02.2017), https://www.sueddeutsche.de/politik/trumps-absage-beim-korrespondenten-dinner-journalisten-mit-politikern-auf-du-und-du-1.3395563 (19.03.2019).

Kellner, Douglas: American Nightmare. Donald Trump, Media Spectacle, and Authoritarian Populism. Amsterdam 2016.

Kolb, Matthias: Trump: Von der Lachnummer zum Horror-Kandidaten. In: Süddeutsche Zeitung (29.04.2016), https://www.sueddeutsche.de/politik/white-house-correspondents-dinner-trumpvon-der-lachnummer-zum-horror-kandidaten-1.2972596 (10.03.2019).

Kreiss, Daniel: The media are about identity, not information. In: Pablo J. Boczkowski/Zizi Papacharissi (Hg.): Trump and the Media. Cambridge, Mass./London 2018: 93-101.

Kriesel, David: Traue keinem Scan, den du nicht selbst gefälscht hast (2014), https://media.ccc. de/v/31c3_-_6558_-_de_-_saal_g_-_201412282300_-_traue_keinem_scan_den_du_nicht_ selbst_gefalscht_hast_-_david_kriesel\#t=135 (13.03.2018).

Latour, Bruno: Die Hoffnung der Pandora. Untersuchungen zur Wirklichkeit der Wissenschaft. Frankfurt a. M. 2002.

Lilla, Mark: The Once and Future Liberal. After Identity Politics. New York 2017.

Luhmann, Niklas: Soziale Systeme. Grundriss einer allgemeinen Theorie. Frankfurt a. M. 2006.

Maihofer, Andrea: Geschlecht als Existenzweise. Macht, Moral, Recht und Geschlechterdifferenz. Frankfurt a. M. 1995. 
Mauss, Marcel: Die Gabe. Die Form und Funktion des Austauschs in archaischen Gesellschaften. Frankfurt a. M. 1968.

McCarthy, Ellen: Calls emerge for a boycott of the White House Correspondents' Association dinner. In: The Washington Post (04.02.2017), https://www.washingtonpost.com/ news/arts-and-entertainment/wp/2017/02/04/wchdboycott/?utm_term=.e37a81c22717 (08.03.2019).

Muñoz, José Esteban: Disidentifications. Queers of Color and the Performance of Politics. Minneapolis/London 1999.

Nagle, Angela: Die digitale Gegenrevolution. Online-Kulturkämpfe der Neuen Rechten von 4chan und Tumblr bis zur Alt-Right und Trump. Bielefeld 2018.

Patterson, Troy: Michelle Wolf and the pseudo-event of the White House Correspondents' Association Dinner. In: The New Yorker (30.04.2018), https://www.newyorker.com/culture/ culture-desk/michelle-wolf-and-the-pseudo-event-of-the-white-house-correspondents-association-dinner (13.03.2019).

Pierce, Charles P.: For my money, Donald Trump has never gotten over this event from 5 years ago. In: Esquire (27.04.2016), https://www.esquire.com/news-politics/politics/news/a44392/ trump-foreign-policy-speech/ (10.03.2019).

Pickard, Victor: When commercialism Trumps democracy: Media pathologies and the rise of the misinformation society. In: Pablo J. Boczkowski/Zizi Papacharissi (Hg.): Trump and the Media. Cambridge, Mass./London 2018: 195-202.

Porter, Marc: Trump's first real tweet was on July 6, 2011. Our theory: It was inspired by Obama. In: The Outline (03.12.2017), https://theoutline.com/post/2445/trump-s-first-real-tweet-wason-july-6-2011?zd=2\&zi=xxp5e7jl (10.03.2019).

Pörksen, Bernhard: Die große Gereiztheit: Wege aus der kollektiven Erregung. München 2018.

Reckwitz, Andreas: Die Gesellschaft der Singularitäten. Zum Strukturwandel der Moderne. Berlin 2017.

Rhodes, Ben: Behind the scenes the day Osama bin Laden was killed (2018), https://lithub.com/ behind-the-scenes-the-day-osama-bin-laden-was-killed/ (22.10.18).

Roswell, Jonathan/Diego-Rosell, Pablo: Explaining nationalist political views: The case of Donald Trump (15.08.2016), https://papers.ssrn.com/sol3/papers.cfm?abstract_id=2822059 (16.04.2019).

Sedgwick, Eve Kosofsky: Epistemology of the Closet. Berkeley/Los Angeles 1990.

Sedgwick, Eve Kosofsky: Paranoid reading and reparative reading; or, your so paranoid, you probably think this introduction is about you. In: Dies. (Hg.): Novel Gazing: Queer Readings in Fiction. Durham 1997: 1-37.

Seeßlen, Georg: Trump! POPulismus als Politik. Berlin 2017.

South Park: PC Principle final justice (Staffel 19, Episode 10), southpark.de (2015), http://www. southpark.de/alle-episoden/s19e10-pc-principal-final-justice\#source $=e 3748950-6 \mathrm{c} 2 \mathrm{a}-4201$ 8e45-89e255c06df1:19eabca7-b00f-4a06-ac66-c9529cef2834\&position=10\&sort=!airdate (13.03.2019).

Steyerl, Hito: White Cube und Black Box. Die Farbmetaphysik des Kunstbegriffs. In: Susan Arndt/Maureen Maisha Eggers/Grada Kilomba/Peggy Piesche (Hg.): Mythen, Masken und Subjekte. Kritischer Weißseinsforschung in Deutschland. Münster 2017: 135-143.

Strick, Simon: Wenn sexuelle Belästiger sich entschuldigen: Monologe, Solipsismus, Terror. In: Gender-Blog der Zeitschrift für Medienwissenschaft (27.05.2018), https://www.zfmedienwissenschaft.de/online/blog/sexuelle_Bel\%C3\%A4stiger_Entschuldigungn (22.11.2018).

Tißberger, Martina/Dietze, Gabriele/Hrzán, Daniela/Husmann-Kastein, Jana (Hg.): Weiß Weißsein - Whiteness. Kritische Studien zu Gender und Rassismus. Frankfurt a. M. u. a 2009.

Trump, Donald: The Correspondents' Dinner is dead (2018a), www.trumptwitterarchive.com/ archive.

Trump, Donald: The White House Correspondents' Dinner was a failure last year (2018b), www. trumptwitterarchive.com/archive.

Trump, Donald/Schwartz, Tony: The Art of the Deal. New York 1987. 
Trump, Donald/Bohner, Kate: The Art of the Comeback. New York 1997.

Tufekci, Zeynep: How social media took us from Tahrir Square to Donald Trump. In: MIT Technology Review (14.08.2018), https://www.technologyreview.com/s/611806/how-socialmedia-took-us-from-tahrir-square-to-donald-trump/ (10.03.2019).

Turner, Fred: Trump on Twitter. How a medium designed for democracy became an authoritarians's mouthpiece. In: Pablo J. Boczkowski/Zizi Papacharissi (Hg.): Trump and the Media. Cambridge, Mass. 2018: 143:150.

Vogl, Joseph: Grinsen ohne Katze. Vom Wissen virtueller Objekte. In: Hans-Christian von Herrmann/Matthias Middell (Hg.): Orte der Kulturwissenschaft: fünf Vorträge. Leipzig 1998: $41-55$.

Washington Post Staff: Full text: Donald Trump announces a presidential bid. In: The Washington Post (16.06.2015), https://www.washingtonpost.com/news/post-politics/wp/2015/06/16/ full-text-donald-trump-announces-a-presidential-bid/?utm_term $=. c 1$ ba $68 \mathrm{~d} 4 \mathrm{~b} 14 \mathrm{c}$ (10.07.2019).

Washington Post Staff: Transcript: Read Michelle Obama's full speech from the 2016 DNC. In: The Washington Post (26.06.2016), https://www.washingtonpost.com/news/post-politics/wp/2016/07/26/transcript-read-michelle-obamas-full-speech-from-the-2016-dnc/?utm_ term $=.44$ b06edd12bd (11.03.2019).

Winkler, Hartmut: Prozessieren. Die dritte, vernachlässigte Medienfunktion. Paderborn 2015.

Woodward, Bob: Furcht. Trump im Weißen Haus. Hamburg 2018.

Open Access Dieses Kapitel wird unter der Creative Commons Namensnennung 4.0 International Lizenz (http://creativecommons.org/licenses/by/4.0/deed.de) veröffentlicht, welche die Nutzung, Vervielfältigung, Bearbeitung, Verbreitung und Wiedergabe in jeglichem Medium und Format erlaubt, sofern Sie den/die ursprünglichen Autor(en) und die Quelle ordnungsgemäß nennen, einen Link zur Creative Commons Lizenz beifügen und angeben, ob Änderungen vorgenommen wurden.

Die in diesem Kapitel enthaltenen Bilder und sonstiges Drittmaterial unterliegen ebenfalls der genannten Creative Commons Lizenz, sofern sich aus der Abbildungslegende nichts anderes ergibt. Sofern das betreffende Material nicht unter der genannten Creative Commons Lizenz steht und die betreffende Handlung nicht nach gesetzlichen Vorschriften erlaubt ist, ist für die oben aufgeführten Weiterverwendungen des Materials die Einwilligung des jeweiligen Rechteinhabers einzuholen. 